\title{
BILINEAR INTEGRATION AND APPLICATIONS TO OPERATOR AND SCATTERING THEORY
}

\author{
BRIAN JEFFERIES
}

\begin{abstract}
We show how to integrate operator valued functions with respect to a spectral or orthogonally scattered measure. Such measures typically have a variation which has either the value zero or infinity on any set and cannot therefore be treated by the approaches of Bartle or Dobrakov. Bilinear integrals of this type arise from trace class operators between Banach function spaces and in the connection between stationary-state scattering theory and time-dependent scattering theory in Hilbert space.
\end{abstract}

1. Introduction. In a fundamental work of mathematical physics [1], Amrein, Georgescu and Jauch establish the connection between stationary-state scattering theory and time-dependent scattering theory by appealing to an interchange in the order of certain operator valued integrals. The study of bilinear integration originated with the work of Bartle [2], but in the foundational paper [1] on the connection between the two approaches to quantum scattering, the integrals were defined as a type of operator valued Riemann integral because they do not fit into Bartle's scheme as described in [6, Chapter II], or even more sophisticated later approaches to bilinear integration $[\mathbf{7}, \mathbf{8}]$. The essential difficulty may be described as follows.

Suppose that $(\mathcal{H},(\cdot \mid \cdot))$ is a Hilbert space and $P: \mathcal{B}(\mathbb{R}) \rightarrow \mathcal{L}(\mathcal{H})$ is the spectral measure associated with a selfadjoint operator $A$ : $\mathcal{D}(A) \rightarrow \mathcal{H}$ with domain $\mathcal{D}(A)$. By this, we mean that $P$ has values in the bounded selfadjoint projection operators and is $\sigma$-additive for the strong operator topology of $\mathcal{L}(\mathcal{H}), P(\mathbb{R})=\mathrm{Id}, P(A \cap B)=P(A) P(B)$

2010 AMS Mathematics subject classification. Primary 28A25, 46A32, Secondary 46N50.

Keywords and phrases. Bilinear integral, trace class operator, nuclear operator, trace, spectral measure.

Received by the editors on April 15, 2013, and in revised form on April 30, 2014. DOI: 10.1216/RMJ-2016-46-1-189 
for every $A, B \in \mathcal{B}(\mathbb{R})$ and the identity

$$
A=\int_{\mathbb{R}} \lambda d P(\lambda)
$$

is valid [19]. The unique spectral measure $P$ satisfying (1.1) is concentrated on the spectrum $\sigma(A) \subseteq \mathbb{R}$ of the selfadjoint operator $A$, that is, $P(B)=0$ for every Borel subset $B$ of $\mathbb{R}$ disjoint from $\sigma(A)$. For any vector $h \in \mathcal{H}$ and pairwise disjoint Borel subsets $B_{1}, B_{2}, \ldots$ of $\mathbb{R}$ we have

$$
\begin{aligned}
\left\|P\left(\cup_{n=1}^{\infty} B_{n}\right) h\right\|_{\mathcal{H}}^{2} & =\left(P\left(\cup_{n=1}^{\infty} B_{n}\right) h \mid P\left(\cup_{n=1}^{\infty} B_{n}\right) h\right) \\
& =\sum_{n, m=1}^{\infty}\left(P\left(B_{n}\right) h \mid P\left(B_{m}\right) h\right) \\
& =\sum_{n, m=1}^{\infty}\left(P\left(B_{n} \cap B_{m}\right) h \mid h\right) \\
& =\sum_{n=1}^{\infty}\left\|P\left(B_{n}\right) h\right\|_{\mathcal{H}}^{2} .
\end{aligned}
$$

Hence, the $\mathcal{H}$-valued measure $P h: B \mapsto P(B) h, B \in \mathcal{B}(\mathbb{R})$, necessarily has finite 2-variation.

On the other hand, the $L^{2}([0,1])$-valued measure $m: B \mapsto \chi_{B}$, $B \in \mathcal{B}([0,1])$, has the property that

$$
\sum_{n=1}^{\infty}\|m((1 /(n+1), 1 / n])\|_{2}=\sum_{n=1}^{\infty} \frac{1}{\sqrt{n(n+1)}}=\infty,
$$

so that $m$ has infinite variation in $L^{2}([0,1])$. It is not hard to see that the variation $V(m): \mathcal{B}(\mathbb{R}) \rightarrow[0, \infty]$ of $m$ defined by

$$
V(m)(B)=\sup _{\mathcal{P} \in \Pi(B)} \sum_{E \in \mathcal{P}}\|m(E)\|_{2}
$$

has values that are either zero or infinity. Here $\Pi(B)$ denotes the set of all finite partitions of $B$ into Borel sets.

The $L^{2}([0,1])$-valued measure $m$ is equal to $Q \mathbf{1}$ for the spectral measure $Q: \mathcal{B}(\mathbb{R}) \rightarrow \mathcal{L}\left(L^{2}([0,1])\right)$ such that $Q(B)$ is the selfadjoint projection operator $h \mapsto \chi_{B} \cdot h, h \in L^{2}([0,1])$, for each $B \in \mathcal{B}(\mathbb{R})$ and 1 is the constant function on the interval $[0,1]$ equal to 1 . The 
spectral measure $Q$ is the spectral measure of the position operator of a quantum particle on a line. Any spectral measure $P$ in a Hilbert space $\mathcal{H}$ is unitarily equivalent to a spectral measure like $Q$, so like the vector measure $m$, the variation $V(P h)$ will have values that are either zero or infinity for $h \in \mathcal{H}$, except in trivial cases.

In scattering theory [1], there is an operator valued function $f$ : $\mathbb{R}_{+} \times \mathbb{R} \rightarrow \mathcal{L}(\mathcal{H})$ and a selfadjoint spectral measure $P: \mathcal{B}(\mathbb{R}) \rightarrow \mathcal{L}(\mathcal{H})$ with respect to which it is necessary to establish the equality of the integrals

$$
\int_{0}^{\infty}\left(\int_{\mathbb{R}} f(t, \sigma) d(P h)(\sigma)\right) d t
$$

and

$$
\int_{\mathbb{R}}\left(\int_{0}^{\infty} f(t, \sigma) d t\right) d(P h)(\sigma)
$$

for any vector $h \in \mathcal{H}$. Denote the Lebesgue measure on $\mathbb{R}$ by $\lambda$. Using Fubini's strategy, our aim is to prove their equality with the integral

$$
\int_{\mathbb{R}_{+} \times \mathbb{R}} f d(\lambda \otimes(P h)) .
$$

The measure $\lambda \otimes(P h): C \times B \mapsto \lambda(C)(P h)(B), B \in \mathcal{B}(\mathbb{R}), C \in \mathcal{B}_{f}\left(\mathbb{R}_{+}\right)$ (Borel subsets of $\mathbb{R}_{+}$with finite Lebesgue measure) is $\mathcal{H}$-valued and the values of the operator valued function $f: \mathbb{R}_{+} \times \mathbb{R} \rightarrow \mathcal{L}(\mathcal{H})$ act on the values of the $\mathcal{H}$-valued measure $\lambda \otimes(P h)$-here lies the pervasive difficulty, even in Hilbert space.

Now let us consider the abstract setting in which $(\Omega, \mathcal{S})$ is a measurable space and $X, Y$ Banach spaces. A $\sigma$-additive map $m: \mathcal{S} \rightarrow X$ is called an $X$-valued vector measure, that is, for all pairwise disjoint sets $B_{n} \in \mathcal{S}, n=1,2, \ldots$, the sum $\sum_{n=1}^{\infty} m\left(B_{n}\right)$ converges in the norm of $X$ and

$$
m\left(\bigcup_{n=1}^{\infty} B_{n}\right)=\sum_{n=1}^{\infty} m\left(B_{n}\right) .
$$

Given an operator valued function $f: \Omega \rightarrow \mathcal{L}(X, Y)$, leaving measurability conditions aside for the moment, we wish to consider the integral $\int_{\Omega} f d m \in Y$ in generality sufficient to treat scattering theory. 
For an element $y^{*} \in Y^{*}$ of the space $Y^{*}$ dual to $Y$, the $X^{*}$-valued function $y^{*} \circ f: \Omega \rightarrow X^{*}$ is defined by

$$
\left\langle x,\left(y^{*} \circ f\right)(\omega)\right\rangle=\left\langle f(\omega) x, y^{*}\right\rangle, \quad x \in X, \omega \in \Omega .
$$

It is reasonable to expect that the identity

$$
\left\langle\int_{\Omega} f d m, y^{*}\right\rangle=\int_{\Omega}\left\langle y^{*} \circ f, d m\right\rangle
$$

ought to hold for each $y^{*} \in Y^{*}$, in which the right-hand side is the integral of the $X^{*}$-valued function $y^{*} \circ f$ acting on the range of the $X$ valued measure $m$. However, the total variation $V(m)(\Omega)$ of $m$ satisfies the equation

$$
V(m)(\Omega)=\sup _{\|s\|_{\infty} \leq 1} \int_{\Omega}\langle s, d m\rangle
$$

by the Hahn-Banach theorem. The supremum is taken over all $X^{*}$ valued $\mathcal{S}$-simple functions $s=\sum_{k=1}^{n} x_{k}^{*} \chi_{B_{k}}$ with $\left\|x_{k}^{*}\right\| \leq 1$ and pairwise disjoint $B_{k} \in \mathcal{S}$ for $k=1, \ldots, n$ and $n=1,2, \ldots$, where

$$
\int_{\Omega}\langle s, d m\rangle=\sum_{k=1}^{n} x_{k}^{*}\left(m\left(B_{k}\right)\right) .
$$

As mentioned above, in the case that $m=P h$ for a spectral measure $P$, the variation $V(m)$ may have only the values 0 and $\infty$. Because there may be so few sets on which $m$ has finite variation, neither of the approaches [2] nor [7] is applicable to $m$.

For spectral measures and their application to scattering theory, we may instead adopt the features outlined in [13] concerning bilinear integration in tensor products. In this approach, we may consider $\int_{\Omega} f \otimes d m \in \mathcal{L}(X, Y) \widehat{\otimes}_{\tau} X$ for some suitable tensor product topology $\tau$ for which the product map $J: \mathcal{L}(X, Y) \otimes X \rightarrow Y$ defined by

$$
J\left(\sum_{j=1}^{n} T_{j} \otimes x_{j}\right)=\sum_{j=1}^{n} T_{j} x_{j}
$$

has a continuous extension $\widehat{J}$ from some completion $\mathcal{L}(X, Y) \widehat{\otimes}_{\tau} X$ into $Y$. Then we may define

$$
\int_{\Omega} f d m:=\widehat{J}\left(\int_{\Omega} f \otimes d m\right) .
$$


For an $\mathcal{L}(X, Y)$-valued $\mathcal{S}$-simple function $f=\sum_{k=1}^{n} T_{k} \chi_{B_{k}}$, we have

$$
\int_{\Omega} f d m:=\widehat{J}\left(\int_{\Omega} f \otimes d m\right)=\sum_{k=1}^{n} T_{k}\left(m\left(B_{k}\right)\right) \in Y .
$$

The choice of the tensor product topology $\tau$ needs to be adapted to the problem at hand. For example, the projective tensor product norm is given by

$$
\|u\|_{\pi}=\inf \left\{\sum_{j=1}^{n}\left\|T_{j}\right\| \cdot\left\|x_{j}\right\|: u=\sum_{j=1}^{n} T_{j} \otimes x_{j}\right\} .
$$

Every element $u \in \mathcal{L}(X, Y) \widehat{\otimes}_{\pi} X$ has a representation

$$
u=\sum_{j=1}^{\infty} T_{j} \otimes x_{j}, \quad T_{j} \in \mathcal{L}(X, Y), x_{j} \in X, j=1,2, \ldots,
$$

with

$$
\sum_{j=1}^{\infty}\left\|T_{j}\right\| \cdot\left\|x_{j}\right\|<\infty
$$

[22, III.7], so that $\widehat{J} u=\sum_{j=1}^{\infty} T_{j} x_{j} \in Y$.

Now we look at a number of concrete examples in operator and probability theory where the phenomenon under consideration arises.

Example 1.1. Let $Y=\mathbb{R}$ and $X=L^{1}([0,1])$. Lebesgue measure on $[0,1]$ is denoted by $\lambda$. Let $\varphi:[0,1] \times[0,1] \rightarrow \mathbb{R}$ be a real valued measurable function and $m(B)=\chi_{B}$ for every $B \in \mathcal{B}([0,1])$. We suppose that, for almost every $s \in[0,1]$, the function $t \mapsto \varphi(s, t)$, $t \in[0,1]$, is essentially bounded, that is, there exists $M_{s}>0$ such that $|\varphi(s, t)| \leq M_{s}$ for almost every $t \in[0,1]$. Moreover, if

$$
K_{\varphi}=\operatorname{essip}_{t \in[0,1]} \int_{0}^{1}|\varphi(s, t)| d s<\infty
$$

then the linear map $T_{\varphi}: \psi \mapsto \int_{0}^{1} \varphi(\cdot, t) \psi(t) d t, \psi \in L^{1}([0,1])$, is a bounded linear operator on $L^{1}([0,1])$, because by the Fubini-Tonelli theorem, we have

$$
\left\|T_{\varphi} \psi\right\|_{1} \leq \int_{0}^{1} \int_{0}^{1}|\varphi(s, t) \| \psi(t)| d t d s
$$




$$
\begin{aligned}
& =\int_{0}^{1}\left(\int_{0}^{1}|\varphi(s, t)| d s\right)|\psi(t)| d t \\
& \leq K_{\varphi}\|\psi\|_{1}
\end{aligned}
$$

for every $\psi \in L^{1}([0,1])$. According to [5, Theorem II.2.12], a weakly compact linear map acting on $L^{1}([0,1])$ is representable. For example, the identity map on $L^{1}([0,1])$ is not representable because it has the distribution $\delta(s-t)$ as its kernel. The representability of operators defined on $L^{p}$-spaces is discussed in detail in the papers [21].

Suppose that $K_{\varphi}<\infty$ and let $\Phi_{\varphi}:[0,1] \rightarrow L^{\infty}([0,1])$ be the function defined by $\Phi_{\varphi}(s)=[\varphi(s, \cdot)]$ for almost all $s \in[0,1]$ (in [21], $\Phi_{\varphi}$ is called the Halmos function associated with $\varphi)$. We assume that $\Phi_{\varphi}(s)$ is zero for those $s \in[0,1]$ where $\varphi(s, \cdot)$ is not essentially bounded. Here $[\psi]$ denotes the collection all functions equal to the measurable function $\psi$ almost everywhere on $[0,1]$. Because $m: \mathcal{B}([0,1]) \rightarrow L^{1}([0,1])$ and $L^{\infty}([0,1])=L^{1}([0,1])^{*}$, the bilinear integral $\int_{0}^{1} \Phi_{\varphi} d m$ is a real number.

For any product $A=B \times C$ in $[0,1]^{2}=[0,1] \times[0,1]$ of Borel subsets $B, C$ of $[0,1]$, the equality

$$
\int_{0}^{1} \Phi_{\chi_{A}} d m=\left\langle\chi_{B}, \chi_{C}\right\rangle=\lambda(B \cap C)
$$

holds and, for any simple function $s$ on $[0,1]^{2}$ based on product sets, the equality

$$
\int_{0}^{1} \Phi_{s} d m=\int_{0}^{1} s(t, t) d t
$$

is valid. Because $V(m)=\lambda$, if $\Phi_{\varphi}$ is strongly $\lambda$-measurable in the Banach space $L^{\infty}([0,1])$, then there exist simple functions $s_{n}$, $n=1,2, \ldots$, based on product sets such that $\Phi_{s_{n}} \rightarrow \Phi_{\varphi} \lambda$ almost everywhere in $L^{\infty}([0,1])$ and with $\varphi(t, t)=\lim _{n \rightarrow \infty} s_{n}(t, t)$, almost everywhere

$$
\int_{0}^{1} \Phi_{\varphi} d m=\lim _{n \rightarrow \infty} \int_{0}^{1} \Phi_{s_{n}} d m=\int_{0}^{1} \varphi(t, t) d t .
$$

The left hand side of the equation is defined in the manner of Bartle [2], [6, Section 8] or Dobrakov [7]. The result applies to the case where the integral kernel $\varphi$ is continuous almost everywhere in $[0,1]^{2}$. 
Hence, a bounded linear operator $T_{\varphi}: L^{1}([0,1]) \rightarrow L^{1}([0,1])$ with integral kernel $\varphi$ for which the bilinear integral $\int_{0}^{1} \Phi_{\varphi} d m$ exists, may be viewed as a generalized "trace class" operator on $L^{1}([0,1])$. If Lebesgue measure on $[0,1]$ is replaced by counting measure on a finite index set $J \subset[0,1]$, then

$$
\int_{0}^{1} \Phi_{\varphi} d m=\sum_{j \in J} \varphi(j, j)
$$

is just the trace of the matrix $\{\varphi(j, k)\}_{j, k \in J}$.

In the simple case of the Volterra integral operator $T_{\varphi}: L^{1}([0,1]) \rightarrow$ $L^{1}([0,1])$ with $\left(T_{\varphi} f\right)(s)=\int_{0}^{s} f(t) d t, f \in L^{1}([0,1]), 0 \leq s \leq t$, the integral kernel is given by

$$
\varphi(s, t)= \begin{cases}1, & \text { if } t \leq s, \\ 0, & \text { if } t>s,\end{cases}
$$

so that $\Phi_{\varphi}(s)=\chi_{[0, s]}$. The function $\Phi_{\varphi}$ is not essentially separably valued in $L^{\infty}([0,1])$, so it is not strongly $\lambda$-measurable in $L^{\infty}([0,1])$ and $\int_{0}^{1} \Phi_{\varphi} d m$ cannot be defined in the sense of $[\mathbf{2}, \mathbf{7}]$. Using Definition 2.2 below, the value $\int_{0}^{1} \Phi_{\varphi} d m=\frac{1}{2}$ is obtained in [11].

Linear operators lying in the image of the projective tensor product $L^{1}([0,1]) \widehat{\otimes}_{\pi} L^{\infty}([0,1])$ in $\mathcal{L}\left(L^{1}([0,1])\right)$ are called nuclear operators on $L^{1}([0,1])$ in the terminology of Grothendieck [22, III.7]. Such operators are represented as $T_{\varphi}$ with $\varphi$ given by

$$
[\varphi]=\sum_{j=1}^{\infty} \psi_{j} \otimes \phi_{j},
$$

and

$$
\sum_{j=1}^{\infty}\left\|\psi_{j}\right\|_{1}\left\|\phi_{j}\right\|_{\infty}<\infty .
$$

Then $\Phi_{\varphi}$ is $m$-integrable, and

$$
\operatorname{tr}\left(T_{\varphi}\right)=\sum_{j=1}^{\infty}\left\langle\psi_{j}, \phi_{j}\right\rangle=\int_{0}^{1} \Phi_{\varphi} d m=\int_{0}^{1} \varphi(t, t) d t .
$$


Note that, if $\Phi_{\tilde{\varphi}}$ is strongly $\lambda$-measurable in $L^{\infty}([0,1])$ and $\widetilde{\varphi}=\varphi$ almost everywhere on $[0,1]^{2}$, then $\Phi_{\tilde{\varphi}}=\Phi_{\varphi}$ almost everywhere by Fubini's theorem and $\int_{0}^{1} \widetilde{\varphi}(t, t) d t=\int_{0}^{1} \varphi(t, t) d t$, although the diagonal $\{(t, t): t \in[0,1]\}$ is a set of measure zero in $[0,1]^{2}$.

In the next example, we must deal with the situation in which the vector measure $m$ has infinite variation.

Example 1.2. Let $Y=\mathbb{R}, 1<p<\infty$ and $X=L^{p}([0,1])$. Let $\varphi:[0,1] \times[0,1] \rightarrow \mathbb{R}$ be a measurable function and $m(B)=\chi_{B}$ for every $B \in \mathcal{B}([0,1])$. We suppose that, for almost every $s \in[0,1]$, the function $t \mapsto \varphi(s, t), t \in[0,1]$, is an element of $L^{q}([0,1])$ for $1 / p+1 / q=1$, that is, there exists an $M_{s}>0$ such that

$$
\int_{0}^{1}|\varphi(s, t)|^{q} d t \leq M_{s}^{q}
$$

for almost every $s \in[0,1]$. Suppose that

$$
T_{\varphi}: \psi \longmapsto \int_{0}^{1} \varphi(\cdot, t) \psi(t) d t,
$$

$\psi \in L^{p}([0,1])$, is a bounded linear operator on $L^{p}([0,1])$ and $\Phi_{\varphi}(s)=$ $[\varphi(s, \cdot)]$ as above.

If the bilinear integral

$$
\int_{0}^{1} \Phi_{\varphi} d m
$$

existed, we would still expect $T_{\varphi}$ to be a type of "trace class" operator, see [17]. Recall that a bounded linear operator $T$ on a separable Hilbert space $\mathcal{H}$ is called trace class if, for any (some) orthonormal basis $\left\langle e_{j}\right\rangle_{j \in \mathbb{N}}$ of $\mathcal{H}$, we have

$$
\sum_{j=1}^{\infty}\left|\left(T e_{j}, e_{j}\right)\right|<\infty .
$$

If $T$ is a selfadjoint trace class operator, then $T$ is compact and the set

$$
\sigma(T)=\left\{t_{j}: j=1,2, \ldots\right\}
$$


of real eigenvalues of $T$ satisfies

$$
\sum_{j \in \mathbb{N}}\left|t_{j}\right|<\infty
$$

and

$$
\operatorname{tr}(T)=\sum_{j \in \mathbb{N}} t_{j}
$$

is the trace of $T$. For example, in the case $p=2$, the equality

$$
\operatorname{tr}\left(T_{\varphi}\right)=\sum_{j=1}^{\infty}\left(T_{\varphi} \psi_{j}, \psi_{j}\right)=\int_{0}^{1} \Phi_{\varphi} d m=\int_{0}^{1} \varphi(t, t) d t
$$

should hold for any orthonormal basis $\psi_{1}, \psi_{2}, \ldots$ of $L^{2}([0,1])$ whenever

$$
\int_{0}^{1} \Phi_{\varphi} d m
$$

exists.

Unfortunately, for every $1<p<\infty$, the variation $V(m)$ of the $L^{p}$ valued measure $m$ only has values 0 and $\infty$, so neither of the bilinear integrals described in [2] nor [7] is applicable in the present context.

According to a result of Grothendieck, the image of the projective tensor product $L^{2}([0,1]) \widehat{\otimes}_{\pi} L^{2}([0,1])$ in $\mathcal{L}\left(L^{2}([0,1])\right)$ coincides with the space of trace class operators [22, III.7]. The more familiar example of the image of the Hilbert space tensor product $L^{2}([0,1]) \widehat{\otimes}_{2} L^{2}([0,1])$ in $\mathcal{L}\left(L^{2}([0,1])\right)$ coincides with the space of Hilbert-Schmidt operators $T_{\varphi}$ with

$$
\int_{0}^{1} \int_{0}^{1}|\varphi(s, t)|^{2} d s d t<\infty
$$

In [13, Proposition 4.2], it was shown that the bilinear integral

$$
\int_{0}^{1} \Phi_{\varphi} \otimes d m
$$

converges in $L^{2}([0,1]) \widehat{\otimes}_{\pi} L^{2}([0,1])$ if and only if $T_{\varphi}$ is a trace class operator on $L^{2}([0,1])$. It follows that equation $(1.3)$ is valid for $p=2$, provided that we interpret the bilinear integral

$$
\int_{0}^{1} \Phi_{\varphi} d m
$$


as

$$
J\left(\int_{0}^{1} \Phi_{\varphi} \otimes d m\right)
$$

for the product map $J: L^{2}([0,1]) \widehat{\otimes}_{\pi} L^{2}([0,1]) \rightarrow \mathbb{C}$ defined by

$$
J[u]=\sum_{j=1}^{\infty}\left(\psi_{j} \mid \phi_{j}\right)
$$

for $[u]=\sum_{j=1}^{\infty} \psi_{j} \otimes \phi_{j}$ and $\sum_{j=1}^{\infty}\left\|\psi_{j}\right\|_{2}\left\|\phi_{j}\right\|_{2}<\infty$. For any function $v$ equal to $\sum_{j=1}^{\infty} \psi_{j} \otimes \phi_{j}$ wherever the sum converges, it follows that

$$
\int_{0}^{1}|v(t, t)| d t \leq \sum_{j=1}^{\infty} \int_{0}^{1}\left|\psi_{j}(t) \phi_{j}(t)\right| d t \leq \sum_{j=1}^{\infty}\left\|\psi_{j}\right\|_{2}\left\|\phi_{j}\right\|_{2}
$$

by the Cauchy-Schwarz inequality and the trace of $T_{\varphi}$ is well defined.

The next example arises from the theory of stochastic processes but exhibits features similar to integration with respect to spectral measures.

Example 1.3. Let $\lambda$ denote Lebesgue measure on $[0,1]$. Suppose that $(\Omega, \mathcal{S}, \mathbb{P})$ is a probability measure space and $m: \mathcal{B}([0,1]) \rightarrow L^{2}(\mathbb{P})$ is a Gaussian random measure with mean zero and variance $\lambda$. By this, we mean that $m$ is a vector measure such that, for every finite collection $B_{1}, \ldots, B_{n}, n=1,2, \ldots$, of pairwise disjoint Borel subsets of $[0,1]$, the random variables $m\left(B_{1}\right), \ldots, m\left(B_{n}\right)$ are independent, and for every $B \in \mathcal{B}([0,1])$, the element $m(B)$ of $L^{2}(\mathbb{P})$ is a real Gaussian random variable with mean zero and variance $\mathbb{E}\left(m(B)^{2}\right)=\lambda(B)$. Then, for all pairwise disjoint Borel subsets $B_{1}, B_{2}, \ldots$ of $[0,1]$, we have

$$
\sum_{j=1}^{\infty}\left\|m\left(B_{j}\right)\right\|_{2}^{2}=\sum_{j=1}^{\infty} \lambda\left(B_{j}\right)=\lambda\left(\bigcup_{j=1}^{\infty} B_{j}\right)
$$

so that $m$ has finite 2 -variation. Because $\|m(B)\|_{2}=\sqrt{\lambda(B)}$ for every $B \in \mathcal{B}([0,1])$, the variation $V(m)$ of $m$ only has values zero and infinity. 
If $X:[0,1] \times \Omega \rightarrow E$ is an adapted process with values in a UMD Banach space $E$, then there exists $c>0$ such that

$$
\begin{aligned}
c^{-1}\left\|\int_{0}^{1} X d m\right\|_{L^{2}(\mathbb{P}, E)} & \leq\left\|\int_{0}^{1} X \otimes d m\right\|_{L^{2}(\mathbb{P} \otimes \mathbb{P}, E)} \\
& \leq c\left\|\int_{0}^{1} X d m\right\|_{L^{2}(\mathbb{P}, E)} .
\end{aligned}
$$

When the stochastic integral

$$
\int_{0}^{1} X d m
$$

exists in $L^{2}(\mathbb{P}, E)$, then it may be represented as

$$
\int_{0}^{1} X d m=\widehat{J} \int_{0}^{1} X \otimes d m,
$$

where $J: L^{0}(\mathbb{P}, E) \otimes L^{0}(\mathbb{P}) \rightarrow L^{0}(\mathbb{P}, E)$ is the product map

$$
\begin{gathered}
(J(f \otimes g))(\omega)=f(\omega) g(\omega), \quad f \in L^{0}(\mathbb{P}, E), \\
g \in L^{0}(\mathbb{P}), \quad \omega \in \Omega,
\end{gathered}
$$

defined on the space $L^{0}(\mathbb{P}, E)$ of strongly $\mathbb{P}$-measurable $E$-valued functions with the topology of convergence in probability in $E$. By restricting $J$ to the linear space

$$
\mathcal{X}=\left\{\int_{0}^{1} X \otimes d m: X \text { is simple and adapted }\right\},
$$

and taking its continuous extension from the closure $\overline{\mathcal{X}}^{L^{2}(\mathbb{P} \otimes \mathbb{P}, E)}$ of $\mathcal{X}$ in $L^{2}(\mathbb{P} \otimes \mathbb{P}, E)$ into $L^{2}(\mathbb{P}, E)$, we obtain the continuous linear map $\widehat{J}$ above, see $[\mathbf{1 0}]$ for the details.

The examples show that the bilinear integral (1.2) arises naturally in operator theory on Hilbert space. We shall first consider the situation for general Banach spaces in Section 2. In Theorem 2.3, we obtain an analogue of Fubini's theorem for bilinear integrals by a simple application of the scalar case. Trace class operators on a Banach function space are considered in Example 1.2. The bilinear 
trace features in the proof of the Cwikel-Lieb-Rosenblum inequality for dominated semigroups in quantum physics [12].

A toy example of quantum scattering theory from [10, Section 4] is considered in Example 2.5, where only approximation in the uniform operator norm is needed. The example shows the need for a deeper analysis in order to treat unbounded selfadjoint operators.

In Section 3, some results concerning different notions of measurability of operator valued functions are gathered. Measurability in the strong operator topology arises most in applications, but it is not a metrizable topology. The strong operator analogue of Pettis's measurability theorem [5, Theorem II.1.2] is given in Theorem 3.5. It does not seem to be in the literature.

Stationary-state and time-dependent approaches to quantum scattering are related by the application of a type of Fubini theorem for bilinear integrals in Hilbert space. The auxiliary Banach space $E$ is constructed in Section 4, and Theorem 4.5 gives a general result on the interchange of integrals relevant to scattering theory, improving on the results of [9]. The proof of integrability in $E$ requires certain technical facts concerning projective tensor products due to Grothendieck [22, Section III.6].

2. Bilinear integration. Let $X, Y$ be Banach spaces. A locally convex space $E$ is said to be bilinear admissible for $X, Y$ if

(a) $E$ contains the vector space $\mathcal{L}(X, Y) \otimes X$ as a dense subspace,

(b) the composition map $J: \mathcal{L}(X, Y) \times X \rightarrow Y$ defined by

$$
J(T, x)=T x, \quad T \in \mathcal{L}(X, Y), x \in X,
$$

has a continuous linear extension from $\mathcal{L}(X, Y) \otimes X$ to $E$, also denoted by $J$.

(c) For $x \in X, x^{*} \in X^{*}$ and $y^{*} \in Y^{*}$, the linear functional defined by

$$
\begin{gathered}
x \otimes y^{*} \otimes x^{*}: T \otimes u \longmapsto\left\langle T x, y^{*}\right\rangle\left\langle u, x^{*}\right\rangle, \\
T \in \mathcal{L}(X, Y), \quad u \in X,
\end{gathered}
$$

is continuous on $\mathcal{L}(X, Y) \otimes X$ for the relative topology of $E$.

(d) The family of all linear functionals $x \otimes y^{*} \otimes x^{*}$ for $x \in X, x^{*} \in X^{*}$ and $y^{*} \in Y^{*}$ separates points of $E$. 
If $Y=X$, then we merely say $E$ is bilinear admissible for $X$. If $\tau$ is a completely separating tensor product topology [13] on $\mathcal{L}(X, Y) \otimes X$, then we may take $E=\mathcal{L}(X, Y) \widehat{\otimes}_{\tau} X$, the completion of the linear space $\mathcal{L}(X, Y) \otimes X$ in the locally convex topology $\tau$. Sometimes the quasicompletion [15, subsection 23.1] is taken.

Remark 2.1. If the Banach space $X$ has the approximation property [22, Section III.9], then $X \otimes X^{*}$ separates points of the projective tensor product $X^{*} \widehat{\otimes}_{\pi} X[\mathbf{1 6}$, subsection $43.2(12)]$, and this is precisely the property needed to define the trace of a nuclear operator on $X[\mathbf{1 7}]$. In Example 1.2, the separation property is what we need to define the generalized trace

$$
\int_{0}^{1} \Phi_{\varphi} d m
$$

when $X$ is a Banach function space such as $L^{p}([0,1]), 1 \leq p<\infty$.

Definition 2.2. Suppose that the locally convex space $E$ is bilinear admissible for the Banach spaces $X$ and $Y$. Let $(\Omega, \mathcal{S})$ be a measurable space. A function $f: \Omega \rightarrow \mathcal{L}(X, Y)$ is said to be $m$-integrable in $E$ for a vector measure $m: \mathcal{S} \rightarrow X$, if for each $x \in X, x^{*} \in X^{*}, y^{*} \in Y^{*}$, the scalar function $\left\langle f x, y^{*}\right\rangle$ is integrable with respect to the scalar measure $\left\langle m, x^{*}\right\rangle$ and, for each $S \in \mathcal{S}$, there exists an element $(f \otimes m)(S)$ of $E$ such that

$$
\left\langle(f \otimes m)(S), x \otimes y^{*} \otimes x^{*}\right\rangle=\int_{S}\left\langle f x, y^{*}\right\rangle d\left\langle m, x^{*}\right\rangle
$$

for every $x \in X, x^{*} \in X^{*}$ and $y^{*} \in Y^{*}$. by

If $f$ is $m$-integrable in $E$, then $\operatorname{fm}(S) \in Y$ is defined for each $S \in \mathcal{S}$

$$
f m(S)=J((f \otimes m)(S)) .
$$

We also denote $f m(S)$ by

$$
\int_{S} f d m
$$

or

$$
\int_{S} f(\omega) d m(\omega)
$$


In the case where $X$ is the set of scalars, $f m$ is the indefinite (Pettis) integral of a $Y$-valued function with respect to a scalar measure $m$. We shall use the term Bochner integral to distinguish the stronger integration process when $f$ is approximated in the norm of $Y$, see Section 3.

Because the linear space $X \otimes Y^{*} \otimes X^{*}$ separates points of $E$, the vector $(f \otimes m)(S) \in E$ is well defined for each $S \in \mathcal{S}$. The same definition is adopted if $\mathcal{S}$ is generated by the $\delta$-ring $\mathcal{S}_{0}$ and $m: \mathcal{S}_{0} \rightarrow X$ is a vector measure on $\mathcal{S}_{0}$. Definition 2.2 facilitates a simple version of Fubini's theorem in the operator context.

Theorem 2.3. Suppose that the locally convex space $E$ is bilinear admissible for the Banach spaces $X$ and $Y$. Let $(\Omega, \mathcal{S})$ be a measurable space and $(\Gamma, \mathcal{E}, \mu)$ a $\sigma$-finite measure space, and $m: \mathcal{S} \rightarrow X$ a vector measure.

Suppose that $f: \Omega \times \Gamma \rightarrow \mathcal{L}(X, Y)$ is $m \otimes \mu$-integrable in $E$. If

(i) for m-almost all $\omega \in \Omega$, the $\mathcal{L}(X, Y)$-valued function $f(\omega, \cdot)$ is $\mu$-integrable, and

(ii) for $\mu$-almost all $\gamma \in \Gamma$, the $\mathcal{L}(X, Y)$-valued function $f(\cdot, \gamma)$ is $m$ integrable in $E$,

then the function

$$
\omega \longmapsto \int_{\Gamma} f(\omega, \gamma) d \mu(\gamma)
$$

is $m$-integrable in $E$, the function

$$
\gamma \longmapsto \int_{\Omega} f(\omega, \gamma) d m(\omega)
$$

is integrable in $\mathcal{L}(X, Y)$ with respect to $\mu$, and the equalities

$$
\begin{aligned}
\int_{\Omega \times \Gamma} f d(m \otimes \mu) & =\int_{\Omega}\left(\int_{\Gamma} f(\omega, \gamma) d \mu(\gamma)\right) d m(\omega) \\
& =\int_{\Gamma}\left(\int_{\Omega} f(\omega, \gamma) d m(\omega)\right) d \mu(\gamma) .
\end{aligned}
$$

hold. 
Proof. Let

$$
\Phi(\omega)=\int_{\Gamma} f(\omega, \gamma) d \mu(\gamma)
$$

for all $\omega \in \Omega$ for which $f(\omega, \cdot)$ is $\mu$-integrable and $\Phi(\omega)=0$ otherwise. For each $x \in X, y^{*} \in Y^{*}, x^{*} \in X^{*}$ and $S \in \mathcal{S}$, we have

$$
\left\langle\Phi(\omega) x, y^{*}\right\rangle=\int_{\Gamma}\left\langle f(\omega, \gamma) x, y^{*}\right\rangle d \mu(\gamma)
$$

so that

$$
\begin{aligned}
\int_{S}\left\langle\Phi(\omega) x, y^{*}\right\rangle d\left\langle m, x^{*}\right\rangle & =\int_{S}\left(\int_{\Gamma}\left\langle f(\omega, \gamma) x, y^{*}\right\rangle d \mu(\gamma)\right) d\left\langle m, x^{*}\right\rangle \\
& =\left\langle(f \otimes(m \otimes \mu))(S \times \Gamma), x \otimes y^{*} \otimes x^{*}\right\rangle
\end{aligned}
$$

by the scalar version of Fubini's theorem. It follows that $\Phi$ is $m$ integrable in $E$ and

$$
\left.\int_{S} \Phi(\omega) \otimes d m(\omega)=f \otimes(m \otimes \mu)\right)(S \times \Gamma)
$$

hence,

$$
\left.\int_{\Omega} \Phi(\omega) d m(\omega)=J(f \otimes(m \otimes \mu))(\Omega \times \Gamma)\right)=\int_{\Omega \times \Gamma} f d(m \otimes \mu) .
$$

A similar appeal to the scalar version of Fubini's theorem applies to the other iterated integral.

Because we are dealing with vector valued integrals, the existence of integrals (i) and (ii) almost everywhere is not ensured by the integrability of $f$ with respect to the product measure $m \otimes \mu$.

Example 2.4. Let $(\Omega, \mathcal{S}, \mu)$ be a finite measure space. The space of all $\mu$-equivalence classes of $\mathcal{S}$-measurable scalar functions is denoted by $L^{0}(\mu)$. It is equipped with the topology of convergence in $\mu$-measure and vector operations pointwise $\mu$-almost everywhere. Any Banach space $X$ that is a subspace of $L^{0}(\mu)$ with the properties that

(i) $X$ is an order ideal of $L^{0}(\mu)$, that is, if $g \in X, f \in L^{0}(\mu)$ and $|f| \leq|g| \mu$ almost everywhere, then $f \in X$, and

(ii) if $f, g \in X$ and $|f| \leq|g| \mu$ almost everywhere, then $\|f\|_{X} \leq\|g\|_{X}$, 
is called a Banach function space (based on $(\Omega, \mathcal{S}, \mu)$ ). We suppose that $X$ contains constant functions and $m: S \mapsto \chi_{S}, S \in \mathcal{S}$, is $\sigma$-additive in $X$, for example, $X$ is $\sigma$-order continuous, see [18, Corollary 3.6]. If $X$ is reflexive and $\mu$ is non-atomic, then it follows from [18, Corollary 3.23 ] that the values of the variation $V(m)$ of $m$ are either zero or infinity.

Suppose that $\varphi: \Omega \times \Omega \rightarrow \mathbb{C}$ is a jointly measurable function and $T_{\varphi}: X \rightarrow X$ is a bounded linear operator such that

$$
T_{\varphi} f=\int_{\Omega} \varphi(\cdot, t) f(t) d \mu(t)
$$

for a dense set of $f \in X$. Suppose also that $\Phi_{\varphi}(s)=[\varphi(s, \cdot)] \in X^{*}$ for $\mu$-almost all $s \in \Omega$, that is, there exists $K_{s}>0$ such that

$$
\left|\int_{\Omega} \varphi(s, t) f(t) d \mu(t)\right| \leq K_{s}\|f\|_{X}, \quad f \in X .
$$

A bounded operator $T_{\varphi}$ for which there exists a bilinear $\left(X, \mathbb{C}^{*}\right)$ admissible space $E$ such that $\Phi_{\varphi}$ is $m$-integrable in $E$, is a type of generalized trace class operator and

$$
\int_{\Omega} \Phi_{\varphi} d m=J \int_{\Omega} \Phi_{\varphi} \otimes d m
$$

is the trace of $T_{\varphi}$. For example, if $X$ has the approximation property [22, Section III.9] and $E=X^{*} \widehat{\otimes}_{\pi} X$, then $T_{\varphi}$ is a nuclear operator and

$$
\int_{\Omega} \Phi_{\varphi} d m
$$

is actually the trace of $T_{\varphi}$ [17]. There are closed subspaces of $\ell^{p}$, $1 \leq p<\infty, p \neq 2$, without the approximation property. Other examples of $E$-traces are given in [11].

As mentioned in Example 1.1, the value

$$
\int_{0}^{1} \Phi_{\varphi} d m=\frac{1}{2}
$$

is obtained in [11] for the Volterra integral operator $T_{\varphi}$ on $L^{2}([0,1])$ with a careful choice of the auxiliary space $E$. The Volterra integral operator is Hilbert-Schmidt but not trace class on $L^{2}([0,1])$. The 
bilinear integral

$$
\int_{0}^{1} \Phi_{\varphi} d m
$$

is a type of singular integral because the diagonal $\{(t, t): t \in[0,1]\}$ has measure zero in $[0,1]^{2}$, so that the integral

$$
\int_{0}^{1} \varphi(t, t) d t
$$

is not well defined for a general integral kernel $\varphi$ associated with the operator $T_{\varphi}$. The auxiliary space $E$ determines the averaging process of $\varphi$ around the diagonal, and the density of the measure

$$
A \longmapsto \int_{A} \Phi_{\varphi} d m, \quad A \in \mathcal{B}([0,1]),
$$

with respect to Lebesgue measure represents the average $t \mapsto \widetilde{\varphi}(t, t)$ of $\varphi$ around the diagonal.

The second basic example from [10, Section 4] is relevant to the connection between stationary-state and time-dependent scattering theory [1] where $H_{0}$ represents the free Hamiltonian operator and $V$ represents an interaction potential. Attention is first restricted to bounded operators.

Example 2.5. Let $(\mathcal{H},(\cdot \mid \cdot))$ be a separable Hilbert space. Suppose $H_{0}$ and $V$ are bounded selfadjoint operators. Then $H=H_{0}+V$ is also a bounded selfadjoint operator, and the function $f_{\epsilon}: \mathbb{R}_{+} \times \mathbb{R} \rightarrow \mathcal{L}(\mathcal{H})$ defined for $\epsilon>0$ by

$$
f_{\epsilon}(t, \sigma)=e^{i t H} V e^{-i(\sigma-i \epsilon) t}
$$

for $t \geq 0$ and $\sigma \in \mathbb{R}$ is uniformly bounded in $\mathcal{L}(\mathcal{H})$. Let $P$ be the spectral measure associated with the selfadjoint operator $H_{0}$ and $h \in \mathcal{H}$. Lebesgue measure on $\mathbb{R}_{+}$is denoted by $\lambda$. We would like to verify the identities

$$
\int_{\mathbb{R}_{+} \times \mathbb{R}} f_{\epsilon}(t, \sigma) d(\lambda \otimes(P h))(t, \sigma)=\int_{0}^{\infty} e^{-\epsilon t} e^{i t H} V e^{-i t H_{0}} h d t
$$




$$
=\int_{\mathbb{R}}\left(\int_{0}^{\infty} e^{-\epsilon t} e^{i t H} V e^{-i t \sigma} d t\right) d(P h)(\sigma)
$$

that help to establish the connection between stationary-state and time-dependent scattering theory in the case of unbounded selfadjoint operators $H_{0}$ and $V$ [1]. The $\mathcal{H}$-valued measure $\lambda \otimes(P h): \mathcal{S} \rightarrow \mathcal{H}$ is given by

$$
\lambda \otimes(P h)(A \times B)=\lambda(A)(P h)(B), \quad A \in \mathcal{B}_{f}\left(\mathbb{R}_{+}\right), B \in \mathcal{B}(\mathbb{R}) .
$$

Here $\mathcal{B}_{f}\left(\mathbb{R}_{+}\right)$is the collection of Borel subsets of $\mathbb{R}_{+}$with finite Lebesgue measure and $\mathcal{S}$ is the $\delta$-ring generated by the collection $\left\{A \times B: A \in \mathcal{B}_{f}\left(\mathbb{R}_{+}\right), B \in \mathcal{B}(\mathbb{R})\right\}$ of product sets in $\mathbb{R}_{+} \times \mathbb{R}$. We check that the projective tensor product

$$
E=\mathcal{L}(\mathcal{H}) \widehat{\otimes}_{\pi} \mathcal{H}
$$

of $\mathcal{H}$ and $\mathcal{L}(\mathcal{H})$ with the uniform operator norm is bilinear admissible for $\mathcal{H}$ and $f_{\epsilon}$ is $(\lambda \otimes(P h))$-integrable in $E$, with the appropriate modification for integration with respect to a vector measure defined on a $\delta$-ring. Because a Hilbert space $\mathcal{H}$ necessarily has the approximation property [22, Section III.9], $\mathcal{H} \otimes \mathcal{H} \otimes \mathcal{H}$ separates points of $E$ [16, subsection $43.2(12)]$.

Let $B(t)=\{\sigma:(t, \sigma) \in B\}$ be the section at $t \geq 0$ of the Borel subset $B$ of $\mathbb{R}_{+} \times \mathbb{R}$. We check that the function

$$
\Phi_{\epsilon}^{B}: t \longmapsto e^{-\epsilon t}\left(e^{i t H} V\right) \otimes\left(e^{-i t H_{0}} P(B(t)) h\right), \quad t \geq 0,
$$

is Bochner integrable in $E$. The function $t \mapsto P(B(t)) h, t \geq 0$, is strongly measurable so by Lusin's theorem on each interval $[0, T]$, there exists a Borel set $\Sigma$ of arbitrarily large measure on which it is continuous in $\mathcal{H}$. Then $\Phi_{\epsilon}^{B}$ is continuous in $E$ on $\Sigma$ because $H$ and $H_{0}$ are assumed to be bounded selfadjoint operators so that the unitary groups $e^{i t H}$ and $e^{-i t H_{0}}, t \in \mathbb{R}$, are continuous for the uniform operator topology. Consequently, $\Phi_{\epsilon}^{B}$ is strongly measurable in $E$. Because

$$
\begin{aligned}
\int_{0}^{\infty}\left\|\Phi_{\epsilon}^{B}\right\|_{E} d t & \leq \int_{0}^{\infty} e^{-\epsilon t}\left\|e^{i t H} V\right\| \cdot \| e^{-i t H_{0}} P(B(t)) h \mid d t \\
& \leq \frac{\|V\|\|h\|}{\epsilon},
\end{aligned}
$$


it follows that $\Phi_{\epsilon}$ is Bochner integrable in $E$. For every $u, v, w \in \mathcal{H}$, we have

$$
\begin{aligned}
\left\langle\int_{0}^{\infty} \Phi_{\epsilon}^{B} d t, u \otimes v \otimes w\right\rangle & =\int_{0}^{\infty} e^{-\epsilon t}\left(e^{i t H} V u \mid v\right)\left(e^{-i t H_{0}} P(B(t)) h \mid w\right) d t \\
& =\int_{B}\left\langle f_{\epsilon}(t, \sigma), u \otimes v\right\rangle d(\lambda \otimes((P h) \mid w))(t, \sigma) .
\end{aligned}
$$

According to Definition 2.2, the function $f_{\epsilon}$ is $(\lambda \otimes(P h))$-integrable in $E$ and, for every Borel subset $B$ of $\mathbb{R}_{+} \times \mathbb{R}$, we have

$$
\int_{B} f_{\epsilon} d(\lambda \otimes(P h))=\int_{0}^{\infty} \Phi_{\epsilon}^{B} d t .
$$

Furthermore, for each $t \geq 0$, the $\mathcal{L}(\mathcal{H})$-valued function $f_{\epsilon}(t, \cdot)$ is $(P h)$ integrable in $E$ the vector measure $P h$ and, for each $B \in \mathcal{B}(\mathbb{R})$, we have

$$
\int_{B} f_{\epsilon}(t, \sigma) \otimes d(P h)=e^{-\epsilon t} e^{i t H} V \otimes e^{-i t H_{0}} P(B) h \in E,
$$

so that

$$
\begin{aligned}
\int_{\mathbb{R}} f_{\epsilon}(t, \sigma) d(P h)(\sigma) & =J \int_{\mathbb{R}} f_{\epsilon}(t, \sigma) \otimes d(P h) \\
& =e^{-\epsilon t} e^{i t H} V e^{-i t H_{0}} h .
\end{aligned}
$$

For each $\sigma \in \mathbb{R}$, the function $f_{\epsilon}(\cdot, \sigma)$ is Bochner integrable for the uniform norm of $\mathcal{L}(\mathcal{H})$ and the $\mathcal{L}(\mathcal{H})$-valued function

$$
\sigma \longmapsto \int_{0}^{\infty} f_{\epsilon}(t, \sigma) d t, \quad \sigma \in \mathbb{R}
$$

is $(P h)$-integrable in $E$. For every Borel subset $C$ of $\mathbb{R}$, we have

$$
\int_{C}\left(\int_{0}^{\infty} f_{\epsilon}(t, \sigma) d t\right) d(P h)=J \int_{0}^{\infty} \Phi_{\epsilon}^{\mathbb{R}_{+} \times C} d t .
$$

The scalar version of Fubini's theorem and the assumption that $\mathcal{H} \otimes$ $\mathcal{H} \otimes \mathcal{H}$ separates points of the bilinear admissible space $E$ ensure the identity

$$
\int_{\mathbb{R}}\left(\int_{0}^{\infty} f_{\epsilon}(t, \sigma) d t\right) d(P h)=\int_{0}^{\infty}\left(\int_{\mathbb{R}} f_{\epsilon}(t, \sigma) d(P h)\right) d t
$$

relevant to scattering theory. 
We can still make sense of Example 2.5 if $H_{0}$ and $V$ are unbounded operators, but it is clear that the auxiliary space $E=\mathcal{L}(\mathcal{H}) \widehat{\otimes}_{\pi} \mathcal{H}$ will no longer suffice, because the unitary groups $e^{i t H}$ and $e^{-i t H_{0}}, t \in \mathbb{R}$, are only continuous for the strong operator topology-it is too much to expect that the function $\Phi_{\epsilon}^{B}$ defined in the example above will be Bochner integrable in the Banach space $\mathcal{L}(\mathcal{H}) \widehat{\otimes}_{\pi} \mathcal{H}$. We first need to consider the approximation of $\mathcal{L}(\mathcal{H})$-valued functions in the strong operator topology.

3. Measurability for operator valued functions. If the linear map $H_{0}: \mathcal{D}\left(H_{0}\right) \rightarrow \mathcal{H}$ is an unbounded selfadjoint operator with domain $\mathcal{D}\left(H_{0}\right)$ in a separable Hilbert space $\mathcal{H}$, then the unitary group $e^{-i t H_{0}}, t \in \mathbb{R}$, is only continuous for the strong operator topology, so we have to approximate operator valued functions formed from $t \mapsto e^{-i t H_{0}}$ in the strong operator topology rather than the uniform operator topology.

The space $\mathcal{L}_{s}(\mathcal{H})$ of linear operators on $\mathcal{H}$ with the strong operator topology, or the topology of simple convergence, is a locally convex Hausdorff space whose topology is given by the fundamental family of seminorms $p_{h}: T \mapsto\|T h\|_{\mathcal{H}}, T \in \mathcal{L}(\mathcal{H})$, for $h \in \mathcal{H}$. Measurability in such locally convex spaces can be treated by the methods of Saab [20] and Chi [4]. We shall spell out the arguments explicitly in the context of $\mathcal{L}(X, Y)$ with $X$ and $Y$ Banach spaces and $X$ is separable. Then bounded absolutely convex subsets of $\mathcal{L}_{s}(X, Y)$ are metrizable in the relative strong operator topology.

Let $(\Omega, \mathcal{S}, \mu)$ be a $\sigma$-finite measure space. An $\mathcal{S}$-measurable function is called simple if it has finitely many values. A function $f: \Omega \rightarrow E$ with values in a Banach space $E$ is said to be strongly $\mu$-measurable if it is the limit $\mu$-almost everywhere of $E$-valued $\mathcal{S}$-simple functions.

A strongly $\mu$-measurable function $f: \Omega \rightarrow E$ is Bochner $\mu$-integrable in $E$ if and only if

$$
\int_{\Omega}\|f(\omega)\|_{E} d \mu(\omega)<\infty
$$

Equivalently, there exist $E$-valued $\mathcal{S}$-simple functions $s_{j}, j=1,2, \ldots$, converging to $f \mu$ almost everywhere such that

$$
\int_{\Omega}\left\|s_{j}-s_{k}\right\|_{E} d \mu \longrightarrow 0 \quad \text { as } j, k \rightarrow \infty .
$$


Then

$$
\int_{B} f d \mu=\lim _{n \rightarrow \infty} \int_{B} s_{n} d \mu
$$

for each $B \in \mathcal{S}$. In particular, $f$ is Pettis $\mu$-integrable, and the integrals agree [5, Definition II.3.2]. The space $L^{1}(\mu, E)$ of equivalence classes $[f]$ of $E$-valued Bochner $\mu$-integrable functions is a Banach space with the norm

$$
\|[f]\|_{1}=\int_{\Omega}\|f\| d \mu .
$$

Definition 3.1. Let $(\Omega, \mathcal{S}, \mu)$ be a $\sigma$-finite measure space and $X, Y$ Banach spaces. The linear space of all bounded linear operators from $X$ to $Y$ with the topology of strong convergence is written as $\mathcal{L}_{s}(X, Y)$.

(a) A function $f: \Omega \rightarrow \mathcal{L}(X, Y)$ is said to be scalarly $\mu$-measurable in $\mathcal{L}(X, Y)$ if and only if, for every $x \in X, y^{*} \in Y^{*}$, the scalar function $\left\langle f x, y^{*}\right\rangle: \omega \mapsto\left\langle f x, y^{*}\right\rangle, \omega \in \Omega$, is $\mu$-measurable.

(b) A function $f: \Omega \rightarrow \mathcal{L}(X, Y)$ is said to be strongly $\mu$-measurable in $\mathcal{L}_{s}(X, Y)$ if and only if there exist $\mathcal{L}(X, Y)$-valued $\mathcal{S}$-simple functions $s_{j}, j=1,2, \ldots$, converging to $f \mu$ almost everywhere in the strong operator topology of $\mathcal{L}(X, Y)$.

(c) A function $f: \Omega \rightarrow \mathcal{L}(X, Y)$ is said to be uniformly $\mu$-measurable in $\mathcal{L}(X, Y)$ if and only if there exist $\mathcal{L}(X, Y)$-valued $\mathcal{S}$-simple functions $s_{j}, j=1,2, \ldots$, converging to $f \mu$ almost everywhere in the uniform operator topology on $\mathcal{L}(X, Y)$.

In Example 2.5, we dealt with operator valued functions which are $\lambda$-measurable in the uniform operator norm of $\mathcal{L}(X, Y)$ with respect to Lebesgue measure $\lambda$ on $\mathbb{R}_{+}$. In practical applications, such measurability is too much to expect. Additional information about spaces of operator valued functions may be found in [3].

Example 3.2. In general, the notions (a), (b) and (c) of measurability of an operator valued function $f: \Omega \rightarrow \mathcal{L}(X, Y)$ need to be distinguished from the requirement that the $X$-valued function $f x: \omega \mapsto f(\omega) x, \omega \in \Omega$, is strongly measurable for each $x \in X$. For example, if $\delta_{s}$ denotes the evaluation map at $s \in[0,1]$ on $X=\ell^{2}([0,1])$, 
and we let $Y=\mathbb{C}$ and $f(t)=\delta_{t}$ for $t \in[0,1]$, then

$$
\int_{0}^{1}|f(t) x| d t=\int_{0}^{1}|x(t)| d t=0
$$

for every $x \in \ell^{2}([0,1])$, because $x(t)=0$ for only countably many $t \in[0,1]$, so $f x:[0,1] \rightarrow \mathbb{C}$ is equal to zero almost everywhere and is certainly measurable.

If $f$ were measurable in either of the senses (b) or (c), then, with respect to Lebesgue measure $\lambda$ on $[0,1]$, we would have $\lambda \circ f^{-1}(\{0\})=1$ by Theorem 3.7 , which is certainly not the case.

The following lemma appeals to an old argument due to Dunford [5, Lemma II.3.1].

Lemma 3.3. Let $(\Omega, \mathcal{S}, \mu)$ be a $\sigma$-finite measure space and $X, Y B a$ nach spaces. Suppose that the operator valued function $f: \Omega \rightarrow$ $\mathcal{L}(X, Y)$ has the property that $f x$ is strongly $\mu$-measurable in $Y$ and

$$
\int_{\Omega}\|f(\omega) x\|_{Y} d \mu(\omega)<\infty, \quad \text { for each } x \in X .
$$

Then, for each $\mu$-measurable subset $B$ of $\Omega$, the $Y$-valued map

$$
x \longmapsto \int_{B} f(\omega) x d \mu(\omega), \quad x \in X,
$$

is a bounded linear operator on $X$ denoted by

$$
\int_{B} f d \mu \text {. }
$$

Proof. The linear map $L: X \rightarrow L^{1}(\mu, Y)$ defined by $L x=f x$, $x \in X$, has the property that, if $x_{n} \rightarrow x$ and $L x_{n} \rightarrow g$ in $L^{1}(\mu, Y)$ as $n \rightarrow \infty$, then $L x_{n_{k}} \rightarrow g$ almost everywhere as $k \rightarrow \infty$ for some subsequence $\left\{x_{n_{k}}\right\}_{k=1}^{\infty}$ of $\left\{x_{n}\right\}_{n=1}^{\infty}$. Because $f(t) x_{n_{k}} \rightarrow f(t) x$ as $k \rightarrow \infty$ for each $t \in[0,1]$, it follows that $g=f x=L x$, so that $L$ is a closed linear map on the Banach space $X$. By the closed graph theorem, $L$ is a continuous linear map and 
$\left\|\int_{B} f(\omega) x d \mu(\omega)\right\| \leq \int_{\Omega}\|f(\omega) x\|_{Y} d \mu(\omega)=\|L x\| \leq\|L\|_{\mathcal{L}\left(X, L^{1}(\mu, Y)\right)}\|x\|$ for every $x \in X$.

It is worthwhile noting when an operator valued function is approximable by operator valued simple functions.

Proposition 3.4. Let $(\Omega, \mathcal{S}, \mu)$ be a $\sigma$-finite measure space and $X, Y$ Banach spaces. Suppose that the operator valued function $f: \Omega \rightarrow$ $\mathcal{L}(X, Y)$ has the property that $f x$ is strongly $\mu$-measurable in $Y$, the $\sigma$-algebra generated by $\{f x: x \in X\}$ is countably generated and

$$
\int_{\Omega}\|f(\omega) x\|_{Y} d \mu(\omega)<\infty, \quad \text { for each } x \in X
$$

Then there exist $\mathcal{L}(X, Y)$-valued $\mathcal{S}$-simple functions $s_{n}, n=1,2, \ldots$, such that for each $x \in X$, we have $s_{n} x \rightarrow f x \mu$ almost everywhere and

$$
\int_{\Omega}\left\|f(\omega) x-s_{n}(\omega) x\right\|_{Y} d \mu(\omega) \rightarrow 0 \quad \text { as } n \rightarrow \infty .
$$

Proof. Let $\Omega_{n} \in \mathcal{S}$ be sets for which $0<\mu\left(\Omega_{n}\right)<\infty$ and $\Omega \backslash \cup_{n=1}^{\infty} \Omega_{n}$ is $\mu$-null. Because $\sigma(\{f x: x \in X\})$ is countably generated, for each $n=1,2, \ldots$, there exist pairwise disjoint subsets $A_{n, j} \in \mathcal{S}, j=1,2, \ldots$. of $\Omega_{n}$, such that

$$
\sigma(\{f x: x \in X\}) \cap \Omega_{n}=\sigma\left(\left\{A_{n, j}: j=1,2, \ldots\right\}\right) .
$$

Let $\mathcal{F}_{n, k}$ be the algebra of all unions of set $A_{n, j}, j=1, \ldots, k$. Then $\mathcal{F}_{n, k}, k=1,2, \ldots$, is an increasing family of finitely generated algebras of subsets of $\Omega_{n}$ such that

$$
\bigvee_{k=1}^{\infty} \mathcal{F}_{n, k}=\sigma(\{f x: x \in X\}) \cap \Omega_{n}
$$

For each $x \in X$ and $n, k=1,2, \ldots$, the conditional expectation $\mathbb{E}\left(f x \mid \mathcal{F}_{n, k}\right)$ with respect to the probability measure $\mu\left(\Omega_{n}\right)^{-1} \mu$ on $\mathcal{S} \cap \Omega_{n}$ 
is the $\mu$-equivalence class of the $\mathcal{S}$-simple function given by

$$
\mathbb{E}\left(f x \mid \mathcal{F}_{n, k}\right)=\sum_{F \in \mathcal{F}_{n, k}} \frac{\int_{F} f x d \mu}{\mu\left(\Omega_{n} \cap F\right)} \chi_{F} .
$$

By the Martingale convergence theorem for vector valued functions [5, Theorem V.2.9], for each $n=1,2, \ldots$, the simple functions $\mathbb{E}\left(f x \mid \mathcal{F}_{n, k}\right)$ converge $f x$ in $Y \mu$ almost everywhere on $\Omega_{n}$ as $k \rightarrow \infty$ and

$$
\int_{\Omega_{n}}\left\|f x-\mathbb{E}\left(f x \mid \mathcal{F}_{n, k}\right)\right\|_{Y} d \mu \rightarrow 0 \quad \text { as } k \rightarrow \infty .
$$

According to Lemma 3.3, we may define the $\mathcal{L}(X, Y)$-valued $\mathcal{S}$ simple function $\mathbb{E}\left(f \mid \mathcal{F}_{n, k}\right)$ by the formula

$$
\mathbb{E}\left(f \mid \mathcal{F}_{n, k}\right)=\sum_{F \in \mathcal{F}_{n, k}} \frac{\int_{F} f d \mu}{\mu\left(\Omega_{n} \cap F\right)} \chi_{F} .
$$

Then $s_{n}=\sum_{k=1}^{n} \mathbb{E}\left(f \mid \mathcal{F}_{n, k}\right), n=1,2, \ldots$, are the required $\mathcal{L}(X, Y)$ valued simple functions.

Next we have a version of Pettis's measurability theorem [5, Theorem II.1.2] for operator valued functions.

Theorem 3.5. Let $(\Omega, \mathcal{S}, \mu)$ be a $\sigma$-finite measure space and $X, Y$ Banach spaces with $X$ separable. Let $\mathcal{D}$ be a countable subset of $X$ whose linear span is dense in $X$. A function $f: \Omega \rightarrow \mathcal{L}(X, Y)$ is strongly $\mu$-measurable in $\mathcal{L}_{s}(X, Y)$ if and only if

(i) $f x$ is $\mu$-essentially separably valued in $Y$ for each $x \in \mathcal{D}$, that is, $(f x)\left(\Omega \backslash N_{x}\right)$ is a norm separable subset of $X$ for some null set $N_{x}, x \in \mathcal{D}$, and

(ii) $f$ is scalarly $\mu$-measurable in $\mathcal{L}(X, Y)$.

Proof. If $f: \Omega \rightarrow \mathcal{L}(X, Y)$ is strongly $\mu$-measurable in $\mathcal{L}_{s}(X, Y)$, then properties (i) and (ii) follow by applying the proof of $[\mathbf{5}$, Theorem II.1.2] to the $Y$-valued function $f x$ for each $x \in X$.

Now suppose that conditions (i) and (ii) hold. By Pettis's measurability theorem in Banach spaces [5, Theorem II.1.2], $f x$ is strongly $\mu$-measurable in $Y$ for each $x \in X$. Because $X$ is separable, there exists a countable dense subset $\mathcal{U}$ of the closed unit ball of $X$. Then 
$\|f(\omega)\|_{\mathcal{L}(X, Y)}=\sup _{x \in \mathcal{U}}\|f(\omega) x\|$ for each $\omega \in \Omega$. It follows that $\|f\|_{\mathcal{L}(X, Y)}$ is a finite $\mathcal{S}$-measurable function and, up to a $\mu$-null set, we can divide $\Omega$ into a countable family of sets $\Gamma$ on which $\|f\|_{\mathcal{L}(X, Y)} \leq c$ for some $c>0$ and $0<\mu(\Gamma)<\infty$.

By Proposition 3.4, there exist $\mathcal{L}(X, Y)$-valued $\mathcal{S}$-simple functions $s_{n}, n=1,2, \ldots$ such that for each $x \in X$, we have $s_{n} x \rightarrow f x \mu$ almost everywhere on $\Gamma$ as $n \rightarrow \infty$ and

$$
\int_{\Gamma}\left\|f(\omega) x-s_{n}(\omega) x\right\|_{Y} d \mu(\omega) \rightarrow 0 .
$$

The set

$$
\Gamma_{0}=\cup_{x \in \mathcal{D}}\left\{\omega \in \Gamma: s_{n}(\omega) x \rightarrow f(\omega) x \text { as } n \rightarrow \infty\right\}
$$

is a set of full measure and, because $f$ is uniformly bounded on $\Gamma$ in the uniform norm of $\mathcal{L}(X, Y)$, it follows that $s_{n}(\omega) \rightarrow f(\omega)$ in $\mathcal{L}_{s}(X, Y)$ as $n \rightarrow \infty$ for every $\omega \in \Gamma_{0}$. We construct $\mathcal{L}(X, Y)$-valued $\mathcal{S}$-simple functions converging to $f \mu$ almost everywhere by piecing together these simple functions. Hence, $f: \Omega \rightarrow \mathcal{L}(X, Y)$ is strongly $\mu$-measurable in $\mathcal{L}_{s}(X, Y)$.

Corollary 3.6. Let $(\Omega, \mathcal{S}, \mu)$ be a $\sigma$-finite measure space and $X, Y$ Banach spaces with $X$ separable. A function $f: \Omega \rightarrow \mathcal{L}(X, Y)$ is strongly $\mu$-measurable in $\mathcal{L}_{s}(X, Y)$ if and only if $f x$ is strongly $\mu$ measurable in $Y$ for each $x \in X$.

Example 3.2 shows that the assumption that $X$ is separable cannot be omitted, even if $Y=\mathbb{R}$.

Let $T$ be a Hausdorff topological space and $(\Omega, \mathcal{S}, \mu)$ a $\sigma$-finite measure space. A function $f: \Omega \rightarrow T$ is called Borel $\mu$-measurable if, for every open subset $U$ of $T$, the set $f^{-1}(U)$ belongs to the $\mu$-completion of $\mathcal{S}$, that is, there exists a $\mu$-null set $N$ such that $f^{-1}(U)=S \Delta N$ for $S \in \mathcal{S}$.

For Banach spaces $X, Y$, the space of bounded linear operators $u: X \rightarrow Y$ endowed with a locally convex topology $\tau$ is written as $\mathcal{L}_{\tau}(X, Y)$. The strong operator topology $s$ is given by the family of seminorms $u \mapsto\|u x\|_{Y}, u \in \mathcal{L}(X, Y)$ for all $x \in X$. 
The topology $\kappa$ of precompact convergence on $\mathcal{L}(X, Y)$ has a neighborhood base of zero consisting of sets $\{u \in \mathcal{L}(X, Y): u(K) \subset U\}$ for all relatively compact subsets $K$ of $X$ and all open neighborhoods $U$ of zero in $Y$.

The weak topology $\sigma$ is given by the family of seminorms $u \mapsto$ $\left|\left\langle u x, y^{*}\right\rangle\right|, u \in \mathcal{L}(X, Y)$ for all $x \in X, y^{*} \in Y^{*}$.

Theorem 3.7. Let $(\Omega, \mathcal{S}, \mu)$ be a finite measure space and $X, Y$ Banach spaces with $X$ separable. The following conditions are equivalent for a function $f: \Omega \rightarrow \mathcal{L}(X, Y)$.

(i) $f$ is strongly $\mu$-measurable in $\mathcal{L}_{s}(X, Y)$.

(ii) $f$ is Borel $\mu$-measurable in $\mathcal{L}_{s}(X, Y)$ and $\mu \circ f^{-1}$ is a radon measure on $\mathcal{L}_{s}(X, Y)$.

(iii) $f$ is Borel $\mu$-measurable in $\mathcal{L}_{\kappa}(X, Y)$ and $\mu \circ f^{-1}$ is a radon measure on $\mathcal{L}_{\kappa}(X, Y)$.

(iv) $f$ is Borel $\mu$-measurable in $\mathcal{L}_{\sigma}(X, Y)$ and $\mu \circ f^{-1}$ is a radon measure on $\mathcal{L}_{\sigma}(X, Y)$.

Proof. Suppose first that (i) holds. Then $f$ is Borel $\mu$-measurable for each of the spaces $\mathcal{L}_{s}(X, Y), \mathcal{L}_{\kappa}(X, Y), \mathcal{L}_{\sigma}(X, Y)$. To see this, observe that $f$ is the limit $\mu$ almost everywhere $\mathcal{S}$-simple functions $s_{n}$, $n=1,2, \ldots$, exist. Because finite subsets are closed sets in each of the topologies, every simple function $s_{n}, n=1,2, \ldots$, is Borel measurable in each of the topologies, and $f$ is the pointwise limit $\mu$-almost everywhere in $\Omega$.

We may replace $Y$ by the closed linear span $Z$ of $\bigcup\left\{f\left(\Omega \backslash N_{x}\right)\right.$ : $x \in \mathcal{D}\}$ for a countable dense subset $\mathcal{D}$ of $X$ and null sets $N_{x} \subset \Omega$ for which the subset $f\left(\Omega \backslash N_{x}\right)$ is separable in $Y$ for $x \in \mathcal{D}$. Then $Z$ is a separable closed subspace $Y$, and $f$ has values in $\mathcal{L}(X, Z)$ off the $\mu$-null set $\bigcup_{x \in \mathcal{D}} N_{x}$, so we suppose that $Y$ is itself separable.

On each set $\Gamma_{c}=\left\{\|f\|_{\mathcal{L}(X, Y)} \leq c\right\}, c>0$, the topologies $s$ and $\kappa$ coincide with pointwise convergence in $Y$ on the countable set $\mathcal{D}$ by the Banach-Steinhaus Theorem. Then the measure $\left(\chi_{\Gamma_{c}} \cdot \mu\right) \circ f^{-1}$ is a finite Borel measure on a complete separable metric space (a Polish space) $\left\{\|u\|_{\mathcal{L}(X, Y)} \leq c\right\}$ for each of the topologies $s$ and $\kappa$, so conditions (ii), (iii) and (iv) hold [23], because the topology $\sigma$ is weaker (coarser) than either $s$ or $\kappa$. 
Now suppose that (iv) holds. Appealing to Theorem 3.5, to prove (i), it suffices to establish that $f x$ has $\mu$-essentially separable range for each $x \in X$. The evaluation map $\delta_{x} u=u x, u \in \mathcal{L}(X, Y)$, is continuous for the weak topology $\sigma$ into $\left(Y, \sigma\left(Y, Y^{*}\right)\right)$ for each $x \in X$, so

$$
\left(\mu \circ f^{-1}\right) \circ \delta_{x}^{-1}=\mu \circ(f x)^{-1}
$$

is a Radon measure for the weak topology $\sigma\left(Y, Y^{*}\right)$ of $Y$. The weak and norm topologies of the Banach space $Y$ are Radon-equivalent [23], so $\mu \circ(f x)^{-1}$ is a Radon measure on $Y$. Its support is contained in the countable union of compact sets, which is norm separable in $Y$, so $f x$ has $\mu$-essentially separable range and condition (i) follows from Theorem 3.5.

Again, Example 3.2 shows that the assumption that $X$ is separable cannot be omitted, even if $Y=\mathbb{R}$.

\section{Remark 3.8.}

(i) On each set $\Gamma_{c}=\left\{\|f\|_{\mathcal{L}(X, Y)} \leq c\right\}$, convergence for the strong operator topology in $\left\{u:\|u\|_{\mathcal{L}(X, Y)} \leq c\right\}$ is determined by the metric

$$
(u, v) \longmapsto \sum_{j=1}^{\infty} 2^{-j}\left\|u x_{j}-v x_{j}\right\|_{Y}, \quad u, v \in \mathcal{L}(X, Y),
$$

for some enumeration $\left\{x_{j}: j=1,2, \ldots\right\}$ of a countable dense subset $\mathcal{U}$ of the closed unit ball of $X$. If $f$ is strongly $\mu$-measurable in $\mathcal{L}_{s}(X, Y)$, then we can use this metric to obtain a version of Lusin's theorem (if $\mu$ is a finite Radon measure) and Egorov's convergence theorem in $\mathcal{L}_{s}(X, Y)$ or $\mathcal{L}_{\kappa}(X, Y)$, if $\mu$ is a finite measure. In a more general context, this idea appeared in [20].

(ii) Suppose that $\mathcal{U}$ is a countable dense subset of the close unit ball of the Banach space $X$. For each $x \in \mathcal{U}$,

$$
\epsilon \mu(\{\|f x\|>\epsilon\}) \leq \int_{\Omega}\|f x\|_{Y} d \mu<\infty
$$

and

$$
\mu(\{\|f\|>0\}) \subseteq \bigcup_{x \in \mathcal{U}} \mu(\{\omega \in \Omega:\|f(\omega) x\|>0\}) .
$$


It follows that for any measure space $(\Omega, \mathcal{S}, \mu)$, the set $\{\|f\|>0\}$ is $\sigma$-finite if

$$
\int_{\Omega}\|f x\|_{Y} d \mu<\infty
$$

for every $x \in X$, provided that $X$ is separable, so in this case, the preceding arguments are valid for an arbitrary measure space.

4. Bilinear integrals in scattering theory. In this section we return to the situation considered in Example 2.5 but with the more physically realistic case of unbounded selfadjoint operators.

Let $(\mathcal{H},(\cdot \mid \cdot))$ be a separable Hilbert space. Suppose $H_{0}: \mathcal{D}\left(H_{0}\right) \rightarrow$ $\mathcal{H}$ and $V: \mathcal{D}(V) \rightarrow \mathcal{H}$ are selfadjoint operators with respective dense domains $\mathcal{D}\left(H_{0}\right) \subset \mathcal{D}(V)$. We suppose that $H=H_{0}+V$ is also a selfadjoint operator on $\mathcal{D}\left(H_{0}\right)$. For example, this is the situation for the free Hamiltonian $H_{0}=-\hbar^{2} /(2 m) \Delta$ of a particle moving in $\mathbb{R}^{3}$ subject to a Coulomb potential $V(x)=c /|x|, x \in \mathbb{R}^{3} \backslash\{0\}, c \neq 0$.

The function $f_{\epsilon}: \mathbb{R}_{+} \times \mathbb{R} \rightarrow \mathcal{L}\left(\mathcal{D}\left(H_{0}\right), \mathcal{H}\right)$ given by

$$
f_{\epsilon}(t, \sigma)=e^{i t H} V e^{-i(\sigma-i \epsilon) t}
$$

for $t \geq 0$ and $\sigma \in \mathbb{R}$ is defined on $\mathcal{D}\left(H_{0}\right)$. Let $P_{0}$ be the spectral measure associated with the selfadjoint operator $H_{0}$ and $h \in \mathcal{D}\left(H_{0}\right)$. As above, the Lebesgue measure on $\mathbb{R}_{+}$is denoted by $\lambda$. The spectral measure $P_{0}$ commutes with the selfadjoint operator $H_{0}$ in the sense that, for each Borel subset $B$ of $\mathbb{R}$, the inclusion $P_{0}(B) \mathcal{D}\left(H_{0}\right) \subset \mathcal{D}\left(H_{0}\right)$ is valid and $H_{0} P_{0}(B) h=P_{0}(B) H_{0} h$ for every $h \in \mathcal{D}\left(H_{0}\right)$. We give $\mathcal{D}\left(H_{0}\right)$ the graph norm

$$
h \longmapsto\left(\|h\|_{\mathcal{H}}^{2}+\left\|H_{0} h\right\|_{\mathcal{H}}^{2}\right)^{1 / 2}, \quad h \in \mathcal{D}\left(H_{0}\right),
$$

under which it is itself a Hilbert space.

Now we seek a suitable space $E$, bilinear admissible for $\mathcal{D}\left(\mathcal{H}_{0}\right), \mathcal{H}$, for which $f_{\epsilon}$ is $(\lambda \otimes(P h))$-integrable in $E$ with respect to the $\mathcal{D}\left(\mathcal{H}_{0}\right)$ valued measure $\lambda \otimes(P h)$.

Let $X, Y$ be Banach spaces. For $y^{*} \in Y^{*}$, we have

$$
\left|\left\langle\sum_{j=1}^{n} T_{j} x_{j}, y^{*}\right\rangle\right|=\left|\left\langle\sum_{j=1}^{n} x_{j}, T_{j}^{*} y^{*}\right\rangle\right| \leq \sum_{j=1}^{n}\left\|x_{j}\right\|_{X} \cdot\left\|T_{j}^{*} y^{*}\right\|_{X^{*}},
$$


for all $T_{j} \in \mathcal{L}(X, Y)$ and $x_{j} \in X, j=1, \ldots, n$ and all $n=1,2, \ldots$ Hence, if we let

$$
\|u\|_{\tau}=\sup _{\left\|y^{*}\right\| \leq 1} \inf \left\{\sum_{j=1}^{n}\left\|x_{j}\right\|_{X} \cdot\left\|T_{j}^{*} y^{*}\right\|_{X^{*}}: u=\sum_{j=1}^{n} T_{j} \otimes x_{j}\right\}
$$

over all representations $u=\sum_{j=1}^{n} T_{j} \otimes x_{j}, n=1,2 \ldots$, of $u \in$ $\mathcal{L}(X, Y) \otimes X$, then the inequality $\|J u\|_{Y} \leq\|u\|_{\tau}$ holds for the product map $J u=\sum_{j=1}^{n} T_{j} x_{j}$ by the Hahn-Banach theorem. The completion of the linear space $\mathcal{L}(X, Y) \widehat{\otimes}_{\tau} X$ with respect to the norm $\|\cdot\|_{\tau}$ is written as $\mathcal{L}(X, Y) \widehat{\otimes}_{\tau} X$.

Lemma 4.1. For each $u=\sum_{j=1}^{n} T_{j} \otimes x_{j} \in \mathcal{L}(X, Y) \otimes X$, let $\mathcal{T}_{u}=$ $\left.\sum_{j=1}^{n} x_{j} \otimes T_{j}^{*} \in \mathcal{L}\left(Y^{*}, X \widehat{\otimes}_{\pi} X^{*}\right)\right)$ denote the linear map $y^{*} \mapsto \sum_{j=1}^{n} x_{j} \otimes$ $\left(T_{j}^{*} y^{*}\right), y^{*} \in Y^{*}$.

Then the linear mapping $k: u \mapsto \mathcal{T}_{u}, u \in \mathcal{L}(X, Y) \otimes X$, is the restriction to $\mathcal{L}(X, Y) \otimes X$ of an isometry of $\mathcal{L}(X, Y) \widehat{\otimes}_{\tau} X$ onto a closed linear subspace of $\left.\mathcal{L}\left(Y^{*}, X \widehat{\otimes}_{\pi} X^{*}\right)\right)$ in the uniform norm.

Proof. For $u \in \mathcal{L}(X, Y) \otimes X$, we have $\|u\|_{\tau}=\left\|\mathcal{T}_{u}\right\|_{\mathcal{L}\left(Y^{*}, X \widehat{\otimes}_{\pi} X^{*}\right)}$.

Lemma 4.2. Let $X, Y$ be Banach spaces with $Y^{*}$ norm separable. If $B$ is a bounded, absolutely convex subset of $\mathcal{L}(X, Y) \otimes X$ with $\tau$-closure $\bar{B}$, then $k \bar{B}$ is a closed subset of $\left.\mathcal{L}_{s}\left(Y^{*}, X \widehat{\otimes}_{\pi} X^{*}\right)\right)$.

Proof. Every element $v$ of the closure of $k \bar{B}$ in $\left.\mathcal{L}_{s}\left(Y^{*}, X \widehat{\otimes}_{\pi} X^{*}\right)\right)$ can be represented as $v=\sum_{j=1}^{\infty} \lambda_{j} x_{j} \otimes T_{j}^{*}$ in $\mathcal{L}_{s}\left(Y^{*}, X \widehat{\otimes}_{\pi} X^{*}\right)$ ), where $\sum_{j=1}^{\infty}\left|\lambda_{j}\right|<\infty$, the finite sum $\sum_{j=1}^{n} \lambda_{j} x_{j} \otimes T_{j}^{*}$ belongs to $B$ for each $n=1,2, \ldots$, and $T_{j}^{*} \rightarrow 0$ in $\mathcal{L}_{s}\left(Y^{*}, X^{*}\right), x_{j} \rightarrow 0$ in $X$ as $j \rightarrow \infty$.

To see this, we note that $Y^{*}$ is separable, so the relative topology of $\left.\mathcal{L}_{s}\left(Y^{*}, X \widehat{\otimes}_{\pi} X^{*}\right)\right)$ on $k \bar{B}$ coincides with the relative topology of $X \widehat{\otimes}_{\pi} F$, where $F$ is the metrizable locally convex space $\mathcal{L}\left(Y^{*}, X^{*}\right)$ endowed with the topology of pointwise convergence on a countable dense subset of the unit ball of $Y^{*}$ [16, (1) page 138]. Then, the given representation for $v$ follows from a result of Grothendieck [22, Theorem III.6.4] applied to $X \widehat{\otimes}_{\pi} F$. 
Because

$$
\begin{aligned}
\left\|\sum_{j=n}^{\infty} \lambda_{j} T_{j} \otimes x_{j}\right\|_{\tau} & \leq \sup _{\left\|y^{*}\right\| \leq 1} \sum_{j=n}^{\infty}\left|\lambda_{j}\right|\left\|x_{j}\right\|_{X}\left\|T_{j}^{*} y^{*}\right\|_{X^{*}} \\
& \leq \sup _{j}\left\{\left\|x_{j}\right\|_{X}\left\|T_{j}\right\|_{\mathcal{L}(X, Y)}\right\} \sum_{j=n}^{\infty}\left|\lambda_{j}\right| \longrightarrow 0
\end{aligned}
$$

as $n \rightarrow \infty$, it follows that $v \in k \bar{B}$, hence $k \bar{B}$ is closed in $\left.\mathcal{L}_{s}\left(Y^{*}, X \widehat{\otimes}_{\pi} X^{*}\right)\right)$.

A Banach space $X$ has the approximation property if $X^{*} \otimes X$ is dense in $\mathcal{L}_{\kappa}(X)$. Each element $\sum_{j=1}^{n} x_{j}^{*} \otimes x_{j}$ of $X^{*} \otimes X$ defines the finite rank operator

$$
x \longmapsto \sum_{j=1}^{n}\left\langle x, x_{j}^{*}\right\rangle x_{j}, \quad x \in X .
$$

Lemma 4.3. The Banach space $E=\mathcal{L}(X, Y) \widehat{\otimes}_{\tau} X$ is bilinear admissible for the Banach spaces $X$ and $Y$, provided that $X$ has the approximation property.

Proof. Properties (a)-(c) of bilinear admissability clearly hold, so it remains to establish property (d). The family of all linear maps $x \otimes y^{*} \otimes x^{*}: T \otimes x \mapsto\left\langle T x, y^{*}\right\rangle\left\langle x, x^{*}\right\rangle$ for $x \in X, x^{*} \in X^{*}$ and $y^{*} \in Y^{*}$ separates points of $E$.

Let $\mathfrak{B}_{e}\left(\left(X \otimes Y^{*}\right)_{s}, X_{s}^{*}\right)$ denote the linear space of all separately continuous bilinear forms on $\left(X \otimes Y^{*}\right) \times X^{*}$ for the topologies of simple convergence $\sigma\left(X \otimes Y^{*}, \mathcal{L}(X, Y)\right)$ and $\sigma\left(X^{*}, X\right)$. It is equipped with the topology $e$ of bi-equicontinuous convergence [16, page 167]. Because $X$ has the approximation property, the canonical linear map

$$
\psi: \mathcal{L}_{s}\left(Y^{*}, X^{*}\right) \widehat{\otimes}_{\pi} X \rightarrow \mathfrak{B}_{e}\left(\left(X \otimes Y^{*}\right)_{s}, X_{s}^{*}\right)
$$

is one-to-one [16, subsection 43.2 (12)].

The topology $\tau$ has a fundamental system of neighborhoods of zero closed for the weaker topology of $\mathcal{L}_{s}\left(Y^{*}, X^{*}\right) \widehat{\otimes}_{\pi} X$, so $E$ embeds in $\mathfrak{B}_{e}\left(\left(X \otimes Y^{*}\right)_{s}, X_{s}^{*}\right)$ too $[\mathbf{1 6}$, subsection $18.4(4)]$. Because $\left(X \otimes Y^{*}\right) \times X^{*}$ separates the space $\mathfrak{B}_{e}\left(\left(X \otimes Y^{*}\right)_{s}, X_{s}^{*}\right)$ of bilinear forms, it follows that $X \otimes Y^{*} \otimes X^{*}$ separates points of $E$. 
Remark 4.4. The approximation property for $X$ is needed to make sense of the integral

$$
\int_{\Omega} f d m
$$

of an $\mathcal{L}(X, Y)$-valued function $f$ with respect to an $X$-valued measure $m$. Similarly, the approximation property is needed to define the trace of a nuclear operator on $X$ in the case $Y=\mathbb{C}$. All Banach spaces of practical interest, including Hilbert spaces, possess the approximation property, see [17].

The following result improves [9, Theorem 4.4] in the sense that we obtain a stronger form of integrability in the conclusion (c) below.

Theorem 4.5. Let $(\mathcal{H},(\cdot \mid \cdot))$ be a separable Hilbert space and $A$ : $\mathcal{D}(A) \rightarrow \mathcal{H}$ a selfadjoint operator with spectral measure $P_{A}$. Let $E=\mathcal{L}(\mathcal{D}(A), \mathcal{H}) \widehat{\otimes}_{\tau} \mathcal{D}(A)$ where the Hilbert space $\mathcal{D}(A)$ is endowed with the graph norm.

Let $(\Gamma, \mathcal{E}, \mu)$ be a $\sigma$-finite measure space. Suppose that the measurable function $u: \mathbb{R} \times \Gamma \rightarrow \mathbb{C}$ has the property that, for every $h \in \mathcal{D}(A)$, the function $u(\cdot, \gamma)$ is $P_{A}$ h-integrable in $\mathcal{D}(A)$.

Let $f: \Gamma \rightarrow \mathcal{L}(\mathcal{D}(A), \mathcal{H})$ be a strongly $\mu$-measurable in $\mathcal{L}_{s}(\mathcal{D}(A) \mathcal{H})$ function for which there exist positive $\mu$-measurable functions $\alpha, \beta, v$, on $\Gamma$, with the following properties:

(i) $|u(\sigma, \gamma)| \leq v(\gamma)$ for every $\sigma \in \mathbb{R}$ and $\gamma \in \Gamma$,

(ii) $\|f(\gamma) h\| \leq \alpha(\gamma)\|A h\|+\beta(\gamma)\|h\|$ for every $h \in \mathcal{D}(A), \gamma \in \Gamma$, and (iii) $\int_{\Gamma} v(\gamma)(\alpha(\gamma)+\beta(\gamma)) d \mu(\gamma)<\infty$.

Then, for each $h \in \mathcal{D}(A)$,

(a) the function

$$
\gamma \longmapsto f(\gamma) \int_{S} u(\sigma, \gamma) d\left(P_{A} h\right)(\sigma), \quad \gamma \in \Gamma,
$$

is Bochner $\mu$-integrable in $\mathcal{H}$ for each $S \in \mathcal{B}(\mathbb{R})$,

(b) the function

$$
\gamma \longmapsto u(\sigma, \gamma) f(\gamma) g, \quad \gamma \in \Gamma
$$

is Bochner $\mu$-integrable in $\mathcal{H}$ for each $\sigma \in \mathbb{R}$ and $g \in \mathcal{D}(A)$, and 
(c) the $\mathcal{L}(\mathcal{D}(A), \mathcal{H})$-valued function

$$
\sigma \longmapsto \int_{T} u(\sigma, \gamma) f(\gamma) d \mu(\gamma), \quad \sigma \in \mathbb{R}
$$

is strongly measurable in $\mathcal{L}(\mathcal{D}(A), \mathcal{H})$ and $\left(P_{A} h\right)$-integrable in $E$ with respect to the $\mathcal{D}(A)$-valued measure $P_{A} h$, for each set $T \in \mathcal{E}$.

Moreover, the equality

$$
\begin{aligned}
& \int_{S}\left(\int_{T} u(\sigma, \gamma) f(\gamma) d \mu(\gamma)\right) d\left(P_{A} h\right)(\sigma) \\
&=\int_{T} f(\gamma)\left(\int_{S} u(\sigma, \gamma) d\left(P_{A} h\right)(\sigma)\right) d \mu(\gamma)
\end{aligned}
$$

holds for every $S \in \mathcal{B}(\mathbb{R})$ and $T \in \mathcal{E}$.

Proof. Property (a) follows from the strong $\mu$-measurability of $f$ in $\mathcal{L}(\mathcal{D}(A), \mathcal{H})$ and the norm estimates

$$
\begin{aligned}
& \int_{\Gamma}\left\|f(\gamma) \int_{S} u(\sigma, \gamma) d\left(P_{A} h\right)(\sigma)\right\| d \mu(\gamma) \\
& \leq \int_{\Gamma} \alpha(\gamma)\left\|A \int_{S} u(\sigma, \gamma) d\left(P_{A} h\right)(\sigma)\right\| d \mu(\gamma) \\
& \quad+\int_{\Gamma} \beta(\gamma)\left\|\int_{S} u(\sigma, \gamma) d\left(P_{A} h\right)(\sigma)\right\| d \mu(\gamma) \\
& \leq\left\|P_{A} h\right\|_{s v(\mathcal{D}(A))} \int_{\Gamma} v(\gamma)(\alpha(\gamma)+\beta(\gamma)) d \mu(\gamma) \\
& <\infty
\end{aligned}
$$

Here $\left\|P_{A} h\right\|_{s v(\mathcal{D}(A))}$ is the semivariation norm [5, page 2] of the $\mathcal{D}(A)$ valued measure $P_{A} h$. The estimates (i)-(iii) also give property (b).

Now let $B$ be an element of the $\sigma$-algebra $\mathcal{B}(\mathbb{R}) \otimes \mathcal{E}$. We check that the $\mathcal{L}(\mathcal{D}(A), \mathcal{H}) \otimes \mathcal{D}(A)$-valued function

$$
\Phi_{B}: \gamma \longmapsto f(\gamma) \otimes \int_{B(\gamma)} u(\sigma, \gamma) d\left(P_{A} h\right)(\sigma), \quad \gamma \in \Gamma
$$


is integrable to the Banach space $E=\mathcal{L}(\mathcal{D}(A), \mathcal{H}) \widehat{\otimes}_{\tau} \mathcal{D}(A)$ and (4.3)

$$
\left\|\int_{\Gamma} \Phi_{B}(\gamma) d \mu(\gamma)\right\|_{\tau} \leq\left\|P_{A} h\right\|_{s v(\mathcal{D}(A))} \int_{\Gamma} v(\gamma)(\alpha(\gamma)+\beta(\gamma)) d \mu(\gamma) .
$$

Firstly, the inequality

$$
\begin{aligned}
\int_{\Gamma} v(\gamma)\left\|f(\gamma)^{*}\right\|_{\mathcal{L}(\mathcal{H}, \mathcal{D}(A))} d \mu(\gamma) & =\int_{\Gamma} v(\gamma)\|f(\gamma)\|_{\mathcal{L}(\mathcal{D}(A), \mathcal{H})} d \mu(\gamma) \\
& =\sup _{\|\psi\|_{\infty} \leq 1} \int_{\Gamma} v(\gamma)\|f(\gamma) \psi(\gamma)\|_{\mathcal{H}} d \mu(\gamma) \\
& \leq \int_{\Gamma} v(\gamma)(\alpha(\gamma)+\beta(\gamma)) d \mu(\gamma)
\end{aligned}
$$

follows from assumption (ii), where the supremum is taken over $\mathcal{D}(A)$ valued $\mathcal{E}$-simple functions $\psi$. It follows that

$$
\int_{\Gamma}\left\|\Phi_{B}(\gamma)\right\|_{\tau} d \mu(\gamma) \leq\left\|P_{A} h\right\|_{s v(\mathcal{D}(A))} \int_{\Gamma} v(\gamma)(\alpha(\gamma)+\beta(\gamma)) d \mu(\gamma) .
$$

We are not assuming that $f$ is strongly $\mu$-measurable for the uniform norm of $\mathcal{L}(\mathcal{D}(A), \mathcal{H})$, so some caution is needed.

Now $k \circ \Phi_{B}: \Gamma \rightarrow \mathcal{L}(\mathcal{D}(A), \mathcal{H} \otimes \mathcal{H})$ defines a linear map $L_{B}: \mathcal{D}(A) \rightarrow$ $L^{1}(\mu, \mathcal{H} \otimes \mathcal{H})$ by

$$
L_{B} g=\left[k \circ \Phi_{B} g\right], \quad g \in \mathcal{D}(A) .
$$

The bounded linear operator $L_{B}$ is the limit in $\mathcal{L}\left(\mathcal{D}(A), L^{1}\left(\mu, \mathcal{H} \widehat{\otimes}_{\pi} \mathcal{H}\right)\right)$ of $\left(P_{n} \otimes I\right) L_{B}, n=1,2, \ldots$, for conditioning operators $P_{n}: L^{1}(\mu, \mathcal{H}) \rightarrow$ $L^{1}(\mu, \mathcal{H}), n=1,2, \ldots$, on finitely generated $\sigma$-algebras, as in the proof of Proposition 3.4. Then $\left(P_{n} \otimes I\right) L_{B}=\left[k \circ \Phi_{B, n}\right]$ for

$$
\Phi_{B, n}: \gamma \longmapsto\left(P_{n} f\right)(\gamma) \otimes \int_{B(\gamma)} u(\sigma, \gamma) d\left(P_{A} h\right)(\sigma), \quad \gamma \in \Gamma,
$$

and $P_{n} f$ is an $\mathcal{L}(\mathcal{D}(A), \mathcal{H})$-valued $\mathcal{E}$-simple function for each $n=$ $1,2, \ldots$, so $\Phi_{B, n}$ has values in $\mathcal{L}_{s}(\mathcal{D}(A), \mathcal{H}) \otimes \mathcal{D}(A)$. Moreover,

$$
\begin{gathered}
\left\|\left(P_{n} \otimes I\right) L_{B}\right\|_{\mathcal{L}\left(\mathcal{D}(A), L^{1}\left(\mu, \mathcal{H} \widehat{\otimes}_{\pi} \mathcal{H}\right)\right)} \leq \int_{\Gamma}\left\|\Phi_{B}\right\|_{\tau} d \mu, \\
\int_{B}\left(P_{n} \otimes I\right) L_{B} d \mu \in \mathcal{L}(\mathcal{D}(A), \mathcal{H}) \otimes \mathcal{D}(A)
\end{gathered}
$$


for each $n=1,2 \ldots$, and

$$
\lim _{n \rightarrow \infty} \int_{C}\left(P_{n} \otimes I\right) L_{B} d \mu=\int_{C} L_{B} d \mu
$$

in $\mathcal{L}_{s}\left(\mathcal{D}(A), \mathcal{H} \widehat{\otimes}_{\pi} \mathcal{H}\right)$ for each $C \in \mathcal{E}$.

We want to show that for each $C \in \mathcal{E}$, the linear map

$$
g \longmapsto \int_{C} L_{B} g d \mu, \quad g \in \mathcal{D}(A),
$$

belongs to the uniform closure $k E$ of $k(\mathcal{L}(\mathcal{D}(A), \mathcal{H}) \otimes \mathcal{D}(A))$ in the space $\mathcal{L}\left(\mathcal{D}(A), \mathcal{H} \widehat{\otimes}_{\pi} \mathcal{H}\right)$ of linear operators. An appeal to Lemma 4.2 and equation (4.4) shows that this is true on any set $\Gamma_{n}=\left\{\left\|\Phi_{B}\right\|_{\tau} \leq n\right\}$ and, because the vector measure $L_{B} \mu: \mathcal{E} \rightarrow \mathcal{L}_{s}\left(\mathcal{D}(A), \mathcal{H} \widehat{\otimes}_{\pi} \mathcal{H}\right)$ has finite variation

$$
V\left(L_{B} \mu\right)(\Gamma)=\int_{\Gamma}\left\|\Phi_{B}(\gamma)\right\|_{\tau} d \mu(\gamma)
$$

in the uniform operator norm of $\mathcal{L}\left(\mathcal{D}(A), \mathcal{H} \widehat{\otimes}_{\pi} \mathcal{H}\right)$, we have $L_{B} \mu: \mathcal{E} \rightarrow$ $k E$. The equality $L_{B} \mu=k \circ\left(\Phi_{B} \cdot \mu\right)$ ensures that $\Phi_{B}$ is $\mu$-integrable in E.

Because the Banach space $E$ is bilinear admissible for $\mathcal{D}(A), \mathcal{H}$, the integral

$$
\int_{B}(u(\sigma, \gamma) f(\gamma)) \otimes d\left(\left(P_{A} h\right) \otimes \mu\right)(\sigma, \gamma) \in E
$$

is uniquely defined by the scalar equation

$$
\begin{aligned}
& \left.\left(\int_{B}(u(\sigma, \gamma) f(\gamma)) \otimes d\left(\left(P_{A} h\right) \otimes \mu\right)\right)(\sigma, \gamma) \mid u \otimes v \otimes w\right) \\
& =\int_{B}(u(\sigma, \gamma)(f(\gamma) u \mid v)) d\left(\left(P_{A} h \mid w\right) \otimes \mu\right)(\sigma, \gamma)=\left(\int_{\Gamma} \Phi_{B} d \mu \mid u \otimes v \otimes w\right)
\end{aligned}
$$

for each $B \in \mathcal{B}(\mathbb{R}) \otimes \mathcal{E}, u, w \in \mathcal{D}(A)$ and $v \in \mathcal{H}$. Moreover, statement (c) holds, and

$$
\begin{aligned}
\int_{S}\left(\int_{T} u(\sigma, \gamma) f(\gamma) d \mu(\gamma)\right) \otimes d\left(P_{A} h\right)(\sigma) \\
=\int_{S \times T}(u(\sigma, \gamma) f(\gamma)) \otimes d\left(\left(P_{A} h\right) \otimes \mu\right)(\sigma, \gamma)
\end{aligned}
$$


for all $S \in \mathcal{B}(\mathbb{R})$ and $T \in \mathcal{E}$. The equality (4.1) of the iterated integrals follows from the scalar version of Fubini's theorem and the bilinear admissability of $E$.

Corollary 4.6. Let $(\mathcal{H},(\cdot \mid \cdot))$ be a separable Hilbert space and $H_{0}$ : $\mathcal{D}\left(H_{0}\right) \rightarrow \mathcal{H}$ be a selfadjoint operator with spectral measure $P_{0}$. Let $E=\mathcal{L}\left(\mathcal{D}\left(H_{0}\right), \mathcal{H}\right) \widehat{\otimes}_{\tau} \mathcal{D}\left(H_{0}\right)$ where the Hilbert space $\mathcal{D}\left(H_{0}\right)$ is endowed with the graph norm.

Suppose that $V: \mathcal{D}(V) \rightarrow \mathcal{H}$ is a selfadjoint operator with dense domain $\mathcal{D}\left(H_{0}\right) \subset \mathcal{D}(V)$. We suppose that $H=H_{0}+V$ is also a selfadjoint operator on $\mathcal{D}\left(H_{0}\right), \epsilon>0$ and the function $f_{\epsilon}: \mathbb{R}_{+} \times \mathbb{R} \rightarrow$ $\mathcal{L}\left(\mathcal{D}\left(H_{0}\right), \mathcal{H}\right)$ is given by

$$
f_{\epsilon}(t, \sigma)=e^{i t H} V e^{-i(\sigma-i \epsilon) t}, \quad t \in \mathbb{R}_{+}, \sigma \in \mathbb{R} .
$$

Then for each $h \in \mathcal{D}\left(H_{0}\right)$,

(a) the function $t \mapsto e^{-\epsilon t} e^{i t H} V e^{-i H_{0} t} g, t \in \mathbb{R}_{+}$, is Bochner integrable in $\mathcal{H}$ for each $g \in \mathcal{D}\left(H_{0}\right)$,

(b) the function $t \mapsto f_{\epsilon}(t, \sigma) g, t \in \mathbb{R}_{+}$, is Bochner $\mu$-integrable in $\mathcal{H}$ for each $\sigma \in \mathbb{R}$ and $g \in \mathcal{D}\left(H_{0}\right)$, and

(c) the $\mathcal{L}\left(\mathcal{D}\left(H_{0}\right), \mathcal{H}\right)$-valued function

$$
\sigma \longmapsto \int_{T} f_{\epsilon}(t, \sigma) d t, \quad \sigma \in \mathbb{R}
$$

is strongly measurable in $\mathcal{L}\left(\mathcal{D}\left(H_{0}\right), \mathcal{H}\right)$ and $\left(P_{0} h\right)$-integrable in $E$ with respect to the $\mathcal{D}\left(H_{0}\right)$-valued measure $P_{0} h$, for each set $T \in$ $\mathcal{B}\left(\mathbb{R}_{+}\right)$.

Moreover, the equality

$$
\int_{S}\left(\int_{T} f_{\epsilon}(t, \sigma) d t\right) d\left(P_{0} h\right)(\sigma)=\int_{T} e^{-\epsilon t} e^{i t H} V e^{-i H_{0} t} P_{0}(S) h d t
$$

holds for every $S \in \mathcal{B}(\mathbb{R})$ and $T \in \mathcal{B}\left(\mathbb{R}_{+}\right)$.

Proof. Statements (a) and (b) follow directly from the facts that $e^{-i t H}$ and $e^{-i t H_{0}}$ are continuous unitary semigroups on $\mathcal{H}$ and $\mathcal{D}\left(H_{0}\right)$. The selfadjoint operator $V$ is closed and has domain containing $\mathcal{D}\left(H_{0}\right)$. By the closed graph theorem it is bounded in the graph norm of the Hilbert space $\mathcal{D}\left(H_{0}\right)$. Conditions (i)-(iii) of Theorem 4.5 are satisfied 
for the function $u(t, \sigma)=e^{-\epsilon t} e^{-i \sigma t}$ and

$$
f: t \longmapsto e^{i t H} V, \quad t \in \mathbb{R}_{+}, \sigma \in \mathbb{R},
$$

where we take $v(t)=e^{-\epsilon t}, t \in \mathbb{R}_{+}$, and $\alpha, \beta$ are constants.

\section{REFERENCES}

1. W.O. Amrein, V. Georgescu and J.M. Jauch, Stationary state scattering theory, Helv. Phys. Acta. 44 (1971), 407-434.

2. R. Bartle, A general bilinear vector integral, Stud. Math. 15 (1956), 337-351.

3. O. Blasco and J. van Neerven, Spaces of operator-valued functions measurable with respect to the strong operator topology, in Vector measures, integration and related topics, Oper. Th. Adv. Appl. 201, Birkhäuser Verlag, Basel, 2010.

4. G.Y.H. Chi, On the Radon-Nikodym theorem in locally convex spaces, in Measure theory, Lect. Notes Math. 541, Springer, Berlin, 1976.

5. J. Diestel and J.J. Uhl Jr., Vector measures, Math. Surv. 15, American Mathematical Society, Providence, 1977.

6. N. Dinculeanu, Vector measures, Inter. Mono. Pure Appl. Math. 95, Pergamon Press, Oxford, 1967.

7. I. Dobrakov, On integration in Banach spaces I, Czech. Math. J. 20 (1970), $511-536$.

8. __ On integration in Banach spaces II, Czech. Math. J. 20 (1970), 680695.

9. L. Garcia-Raffi and B. Jefferies, An application of bilinear integration to quantum scattering, J. Math. Anal. Appl. 415 (2014), 394-421.

10. B. Jefferies, Some recent applications of bilinear integration, in Vector measures, integration and related topics, Oper. Th. Adv. Appl. 201, Birkhäuser Verlag, Basel, 2010.

11. , Lattice trace operators, J. Operators 2014, article ID 629502.

12. Geom., doi 10.1007/ s11040-014-9145-6, 2014.

13. B. Jefferies and S. Okada, Bilinear integration in tensor products, Rocky Mountain J. Math. 28 (1998), 517-545.

14. B. Jefferies and P. Rothnie, Bilinear integration with positive vector measures, J. Aust. Math. Soc. 75 (2003), 279-293.

15. G. Köthe, Topological vector spaces I, Springer-Verlag, Berlin, 1969.

16. , Topological vector spaces II, Springer-Verlag, Berlin, 1979.

17. G.L. Litvinov, Nuclear operators, Encycl. Math., M. Hazewinkel, ed., Springer, New York, 2001.

18. S. Okada, W. Ricker and E. Sánchez Pérez, Optimal domain and integral extension of operators. Acting in function spaces, Oper. Th.: Adv. Appl. 180, Birkhauser Verlag, Basel, 2008. 
19. W. Rudin, Functional analysis, McGraw-Hill, New York, 1973.

20. E. Saab, On the Radon-Nikodym property in a class of locally convex spaces, Pac. J. Math. 75 (1978) 281-291.

21. W. Schachermayer, Integral operators on $L^{p}$ spaces I, II, Indiana Univ. Math. J. 30 (1981), 123-140, 261-266; Addendum to: Integral operators on $L^{p}$ spaces, Indiana Univ. Math. J. 31 (1982), 73-81.

22. H. Schaefer, Topological vector spaces, Grad. Texts Math. 3, Springer-Verlag, Berlin, 1980.

23. L. Schwartz, Radon measures in arbitrary topological spaces and cylindrical measures, Tata Inst. Fund. Research, Oxford University Press, Bombay, 1973.

School of Mathematics, The University of New South Wales, NSW 2052 Australia

Email address: b.jefferies@unsw.edu.au 